\title{
p53/microRNA-214/ULK1 axis impairs renal tubular autophagy in diabetic kidney disease
}

\author{
Zhengwei Ma, ${ }^{1}$ Lin Li, ${ }^{2}$ Man J. Livingston, ${ }^{1}$ Dongshan Zhang, ${ }^{3}$ Qingsheng Mi, ${ }^{4}$ Ming Zhang, ${ }^{1}$ Han-Fei Ding,,${ }^{5}$ Yuqing Huo, ${ }^{6}$ \\ Changlin Mei, ${ }^{2}$ and Zheng Dong ${ }^{1,7}$ \\ 'Department of Cellular Biology and Anatomy, Medical College of Georgia at Augusta University, Augusta, Georgia, USA. Department of Nephrology, Changzheng Hospital, Second Military Medical University, \\ Shanghai, China. ${ }^{3}$ Department of Emergency Medicine, Second Xiangya Hospital at Central South University, Changsha, China. ${ }^{4}$ Center for Cutaneous Biology and Immunology Research, Department of \\ Dermatology, Henry Ford Health System, Detroit, Michigan, USA. ${ }^{5}$ Cancer Center and ${ }^{6}$ Vascular Biology Center, Medical College of Georgia at Augusta University, Augusta, Georgia, USA. ${ }^{7}$ Charlie Norwood VA \\ Medical Center, Augusta, Georgia, USA.
}

\begin{abstract}
Dysregulation of autophagy in diabetic kidney disease (DKD) has been reported, but the underlying mechanism and its pathogenic role remain elusive. We show that autophagy was inhibited in DKD models and in human diabetic kidneys. Ablation of autophagy-related gene 7 (Atg7) from kidney proximal tubules led to autophagy deficiency and worse renal hypertrophy, tubular damage, inflammation, fibrosis, and albuminuria in diabetic mice, indicating a protective role of autophagy in DKD. Autophagy impairment in DKD was associated with the downregulation of unc-51-like autophagyactivating kinase 1 (ULK1), which was mediated by the upregulation of microRNA-214 (miR-214) in diabetic kidney cells and tissues. Ablation of miR-214 from kidney proximal tubules prevented a decrease in ULK1 expression and autophagy impairment in diabetic kidneys, resulting in less renal hypertrophy and albuminuria. Furthermore, blockade of p53 attenuated miR-214 induction in DKD, leading to higher levels of ULK1 and autophagy, accompanied by an amelioration of DKD. Compared with nondiabetic samples, renal biopsies from patients with diabetes showed induction of $\mathrm{p} 53$ and miR-214, associated with downregulation of ULK 1 and autophagy. We found a positive correlation between p53/miR-214 and renal fibrosis, but a negative correlation between ULK1/LC3 and renal fibrosis in patients with diabetes. Together, these results identify the p53/ miR-214/ULK1 axis in autophagy impairment in diabetic kidneys, pinpointing possible therapeutic targets for DKD.
\end{abstract}

\section{Introduction}

Diabetic kidney disease (DKD), a major complication of diabetes mellitus, is the leading cause of chronic kidney disease (CKD) and end-stage renal disease (ESRD). Approximately 30\%-40\% of patients with diabetes mellitus develop DKD and reach ESRD or die before its onset. Traditionally characterized by pathological changes in glomeruli, DKD may involve a critical component of tubulointerstitial injury in its pathogenesis (1-4). At the cellular level, DKD involves altered intracellular metabolism related to hyperglycemia, hemodynamic changes, inflammation, and intracellular stress associated with renal hypoxia, ROS, mitochondrial dysfunction, and ER disruption (1-3, 5-8). Structurally, renal hypertrophy, described as an enlarged kidney, is one of the first changes in DKD due to increases in glomerular and tubular cell sizes. Although hypertrophy is initially compensatory or adaptive, it later participates in the process of maladaptation of renal function, leading to cellular atrophy or degeneration, renal fibrosis, and progression of DKD $(4,9,10)$. Accordingly, patients with diabetes who have renal hypertrophy are more likely to develop ESRD than patients with smaller kidneys (11).

Conflict of interest: The authors have declared that no conflict of interest exists. Copyright: (5) 2020, American Society for Clinical Investigation.

Submitted: December 6, 2019; Accepted: June 17, 2020; Published: August 17, 2020.

Reference information: J Clin Invest. 2020;130(9):5011-5026.

https://doi.org/10.1172/JCl135536.
Autophagy is a highly conserved, catabolic process in which superfluous or damaged organelles as well as protein aggregates are degraded via lysosomes (12). Autophagy is important for cellular homeostasis and function and is also a stress-responsive mechanism in pathophysiological conditions. Dysfunction of autophagy contributes to the pathogenesis of a variety of diseases, ranging from cancer to neurodegeneration to cardiovascular disorders (13). Dysregulation of autophagy has been implicated in DKD (14-16). Podocytes in the glomerulus showed a significant decrease of autophagy in streptozotocin-induced (STZ-induced) diabetic mice (17), and autophagy deficiency in podocytes led to massive proteinuria in high-fat diet-induced diabetic mice (18). Moreover, patients with diabetes who have massive proteinuria showed impairment of podocyte autophagy with podocyte loss, supporting a role of autophagy in maintaining podocyte viability and function in DKD (18). Autophagy dysfunction has also been demonstrated in kidney proximal tubules in DKD. In 1992, Barbosa Junior Ade and colleagues showed that proximal tubule cells in STZ-induced diabetic rats had significantly lower volume and density of autophagic vacuoles, accompanied by the accumulation of autophagy substrates (19). More recent work demonstrated autophagy impairment in kidney tubule cells in obesity-related or type 2 diabetes (20-22). Sakai et al. further suggested that tubular autophagy may become defective in type 1 and type 2 diabetes at late and early stages, respectively (23). Renal tubule cells of human diabetic kidneys showed the accumulation of autophagosomes and reduced autophagic clearance $(24,25)$. 
A

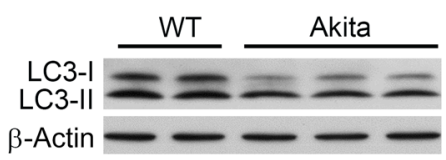

D

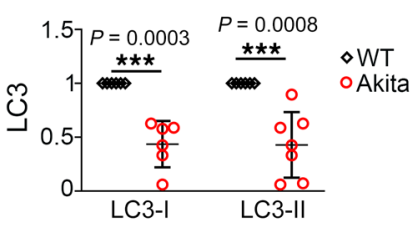

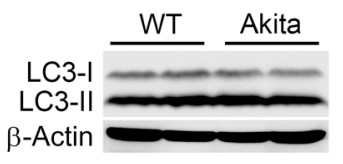
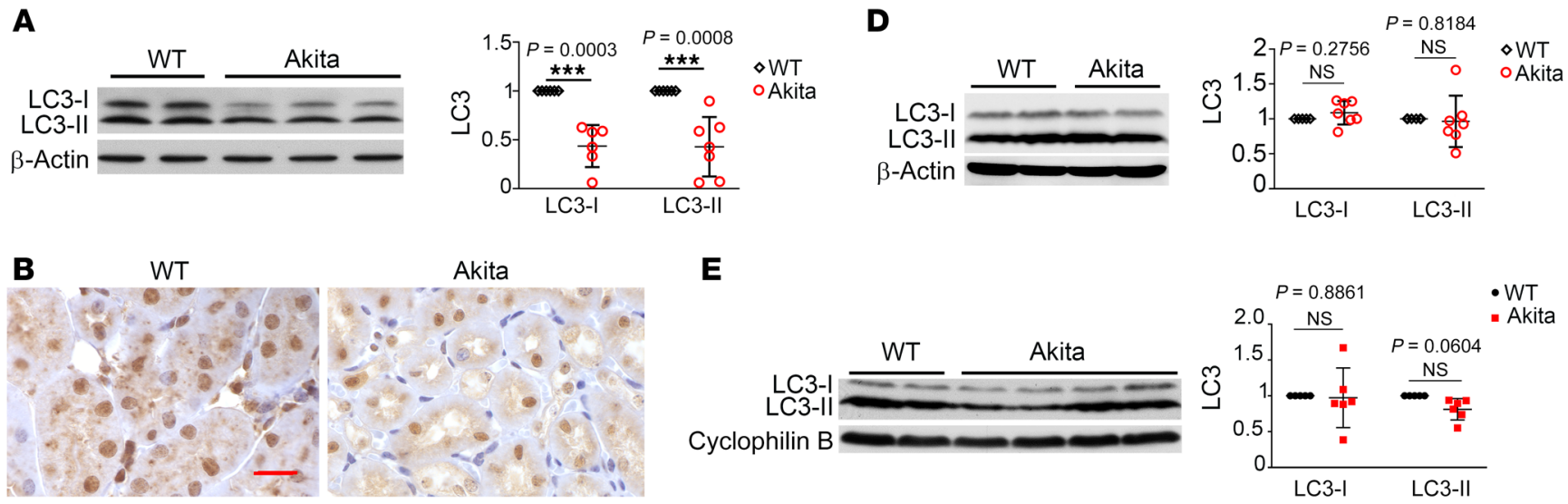

E
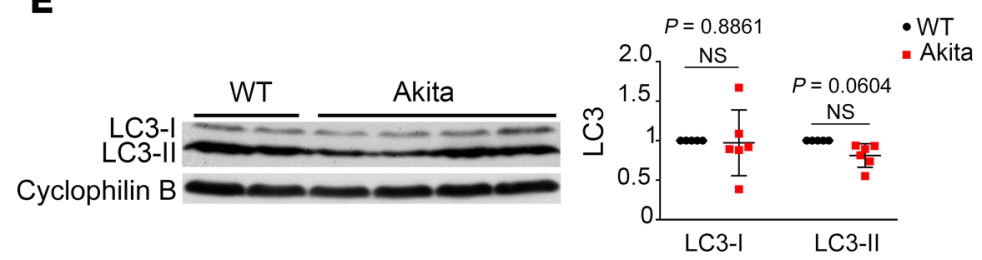

C

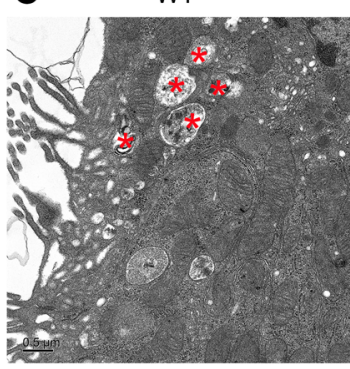

Akita

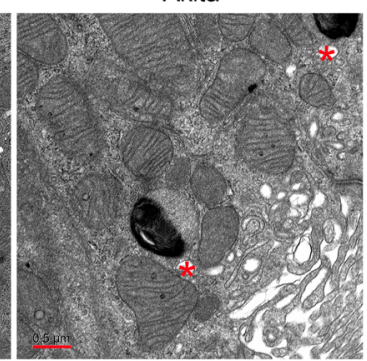

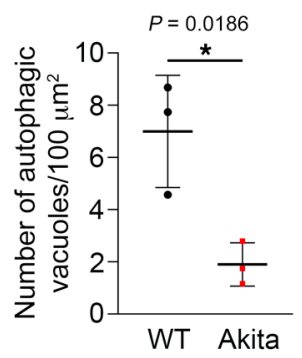

$\mathbf{F}$

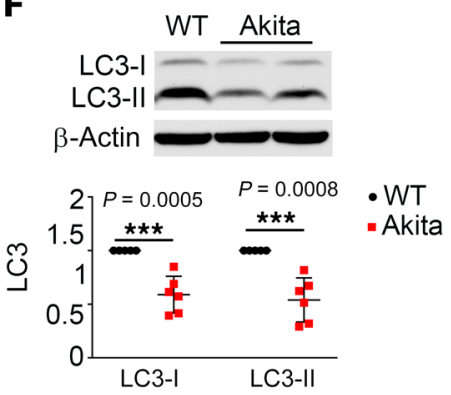

Figure 1. Autophagy in renal tubule cells is decreased in Akita mice. (A) Immunoblots (left) and densitometric analysis (right) showing decreased LC3-I and LC3-II in kidney cortex tissues from 14-week-old Akita mice compared with tissues form nondiabetic WT mice ( $n \geq 6$; 2-tailed Student's $t$ test). (B) IHC staining of LC3 showing a reduced number of LC3 puncta in kidney tubules of Akita mice at 14 weeks. Scale bar: $50 \mu \mathrm{m}$. (C) Representative electron micrographs (original magnification, $\times 10,000$ ) showing a reduced number of autophagic vacuoles in kidney tubule cells in Akita mice at 14 weeks. Asterisks indicate autophagic vacuoles. ( $\mathbf{D}$ and $\mathbf{E}$ ) Immunoblots and densitometric analysis showing no significant changes in LC3-I or LC3-II in kidney cortex samples from 7-week-old (D) and 9-week-old (E) Akita mice ( $n \geq 5$; 2-tailed Student's $t$ test). (F) Immunoblot and densitometric analysis showing decreased LC3-I and LC3-II in kidney cortex samples from 11- to 14-week-old Akita mice ( $n \geq 5 ; 2$-tailed Student's $t$ test). ${ }^{*} P<0.05$ and ${ }^{* * *} P<0.001$.

Thus, despite some variations (26), there is a general recognition that autophagy is impaired in DKD (14-16). However, the significance of autophagy impairment in DKD, especially the association between renal tubular autophagy, renal hypertrophy, and DKD progression, has not been established. Moreover, autophagy regulation and its underlying mechanism in diabetes remain largely unclear. In this study, we demonstrate autophagy dysfunction in renal tubule cells in both experimental models of DKD and kidneys of patients with diabetes. Autophagy impairment in these conditions contributes to renal hypertrophy, related pathologies, and the progression of DKD. Mechanistically, we show that p53 induced miR-214 in DKD, which repressed the key autophagyinitiating protein kinase unc-51-like autophagy-activating kinase 1 (ULK1), resulting in autophagy impairment.

\section{Results}

Autophagy in renal tubule cells is decreased in diabetic mice. To examine autophagy in diabetic kidneys, we first tested Akita mice, a type 1 diabetic model with insulin gene mutation $(27,28)$. Akita mice developed hyperglycemia from approximately 3 weeks of age, with an average fasting blood glucose of $269 \mathrm{mg} / \mathrm{dL}$. At 14 weeks of age, Akita mice had significantly lower levels of LC3-I and LC3-II (biochemical hallmark of autophagy) in kidney tissues compared with levels in WT mice (Figure 1A). Consistently, IHC staining showed that Akita mice had fewer LC3-positive puncta in the cytoplasm of renal tubule cells than did WT mice (Figure 1B). Electron microscopy also revealed fewer autophagic vesicles in the renal tubule cells of Akita mouse kidneys compared with WT mouse kidneys (Figure 1C).

We further monitored the time course of renal autophagy changes during diabetes in Akita mice. At 7 weeks of age, neither Akita mice nor WT mice showed differences in renal LC3 expression (Figure 1D). LC3 expression was lower in Akita mice kidneys at 9 weeks (Figure 1E), but the difference was not statistically significant until 11 weeks (Figure 1F), suggesting a time-dependent decrease of renal autophagy in diabetes.

We also examined renal autophagy in STZ-induced diabetes in C57BL/6 mice, in which diabetes was indicated by a fasting blood glucose level above $280 \mathrm{mg} / \mathrm{dL}$. At 11 weeks, STZ-treated mice showed significantly lower LC3-I and LC3-II expression levels in kidneys compared with levels in control mice (Figure 2A). These mice also had fewer LC3 puncta in kidney tubule cells than did untreated control mice (Figure 2B).

The decrease of autophagy observed in diabetic kidneys might have resulted from changes in autophagosome formation and/or autolysosomal degradation. Thus, we further monitored autophagy dynamics by using CAG-RFP-GFP-LC3-transgenic mice, in which punctate colocalization of red RFP with green GFP fluorescence indicates an autophagosome, whereas an RFP-only punctum without GFP signal indicates an autolysosome, based on 
A

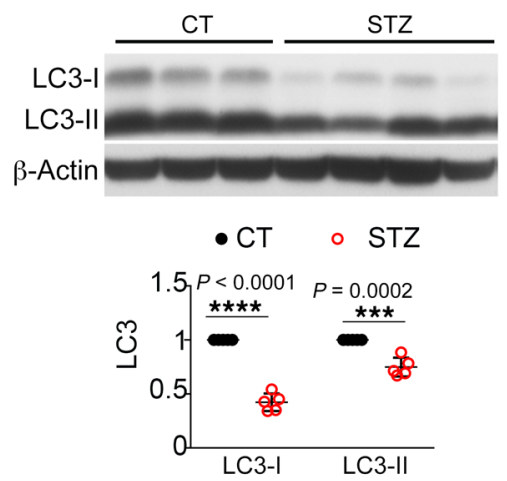

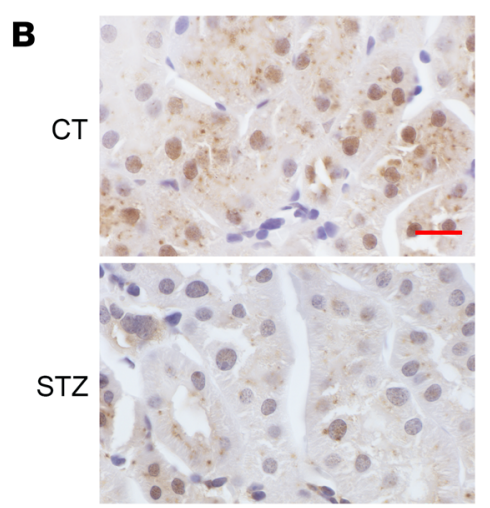

C

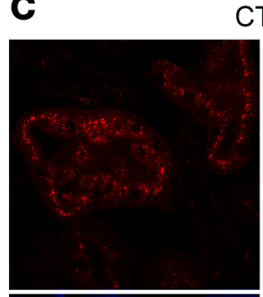

CT
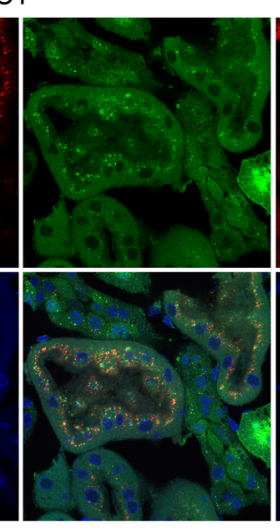

DAPI

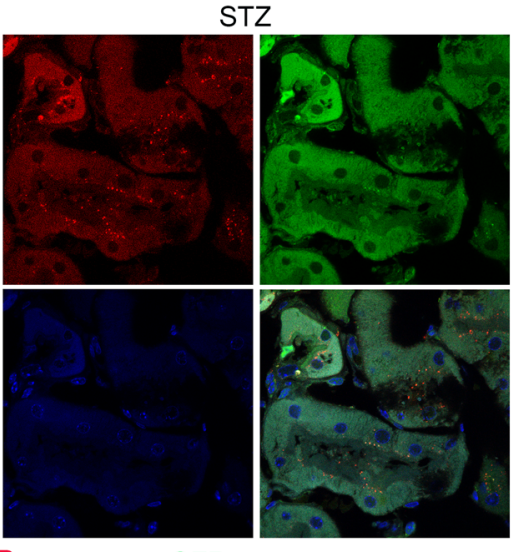

GFP

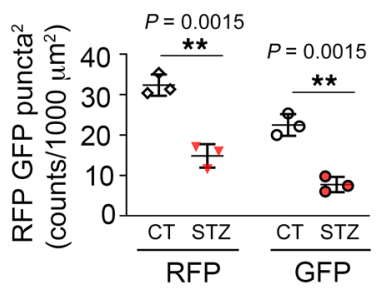

Figure 2. Autophagy in renal tubule cells is decreased in STZ-treated mice. (A) Immunoblots and densitometric analysis showing decreased LC3-I and LC3-II expression in kidney cortex tissues from STZ-treated diabetic C57BL/6 mice ( $n=5$; 2 -tailed Student's $t$ test). (B) IHC staining for LC3 showing a reduced number of LC3 puncta in kidney tubules of STZ-treated mice. Scale bar: $50 \mu \mathrm{m}$. (C) RFP-GFP-LC3 mice were injected with STZ or citrate buffer as a control. Representative images and quantification of GFP-LC3 and RFP-LC3 puncta showing significant decreases in GFP-LC3 and RFP-LC3 puncta in STZ-treated mice $\left(n=3\right.$; 2 -tailed Student's $t$ test). ${ }^{* *} P<0.01,{ }^{* *} P<0.001$, and ${ }^{* * *} P<0.0001$. CT, control.

the fact that acid-sensitive GFP fluorescence is quenched in the low-pH environment ( $\mathrm{pH} 4-5$ ) of autolysosomes, whereas acidinsensitive RFP is more stable and maintained $(29,30)$. As shown in Figure 2C, STZ-treated mice had significantly fewer GFP- and RFP-LC3 puncta in kidney tubule cells than did control animals. In quantification, autophagosomes labeled with GFP dots were reduced from 23 per $1000 \mu^{2}$ tissue in control kidneys to 8 per $1000 \mu \mathrm{m}^{2}$ tissue in diabetic kidneys, whereas autolysosomes that appeared as RFP-only dots were reduced from 10 to 7 per 1000 $\mu \mathrm{m}^{2}$ tissue, indicating an overall autophagy impairment in renal tubules in the diabetic kidneys.

Autophagy deficiency in proximal tubules exaggerates renal hypertrophy and tissue damage in diabetic mice. Autophagy is a cellular process of catabolism or degradation. Thus, we postulated that the autophagy impairment observed in diabetic kidney tubules might contribute to renal hypertrophy, an early pathogenic feature of DKD. To test this, we first determined the effect of autophagy deficiency in kidney proximal tubules in Akita mice. Akita mice were bred with kidney proximal tubule-specific autophagy-related gene 7-knockout (PT-Atg $7^{-/}$) mice (31) to generate PT-Atg7-/Akita mice and PT-Atg $7^{+/+}$Akita mice for comparison (Supplemental Figure 1A; supplemental material available online with this article; https://doi.org/10.1172/JCI135536DS1). Compared with
PT-Atg $7^{+/+}$Akita mice, PT-Atg $7^{-/-}$Akita mice showed lower levels of Atg7 and LC3-II in kidney tissues, validating Atg7 deficiency and autophagy suppression (Supplemental Figure 1B). Compared with WT littermates, both PT- $A \operatorname{tg} 7^{+/+}$and PT-Atg7 $7^{-/}$Akita mice had higher levels of fasting blood glucose and lower body weights (Supplemental Figure 2, A and B). However, PT-Atg7-/- Akita mice had larger kidneys (Supplemental Figure 2C) and a higher kidney/body weight ratio (Figure 3A) than did PT-Atg $7^{+/+}$Akita mice, indicating the development of more severe hypertrophy in diabetic kidneys when tubular autophagy was suppressed. Renal histological analyses revealed that PT- $A \operatorname{tg} 7^{-/}$, but not PT- $A t g 7^{+/+}$, Akita mice had kidney tubule cells with acidophilia (Figure 3B; asterisks indicate acidophilic cells). In addition, PT-Atg $7^{-1-}$ Akita mice showed other signs of renal tubule damage, including lysis of tubular cells and loss of tubular integrity (Figure 3B, arrowheads).

We then examined macrophage infiltration, which would indicate renal inflammation. As shown in Figure 3C, we observed very few macrophages in kidney tissues from nondiabetic WT mice (regardless of Atg7 expression) and in Akita diabetic mice with Atg7 expression. However, PT-Atg7 $7^{-/-}$Akita mice had substantial infiltration of macrophages into the renal interstitium (arrowheads), suggesting that autophagy deficiency in proximal tubules promoted renal inflammation during diabetes. We also 
A
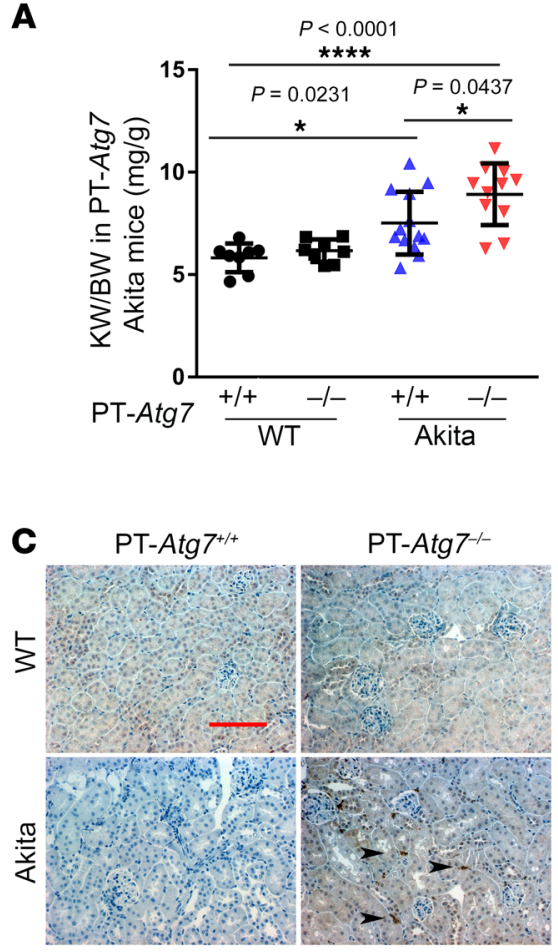
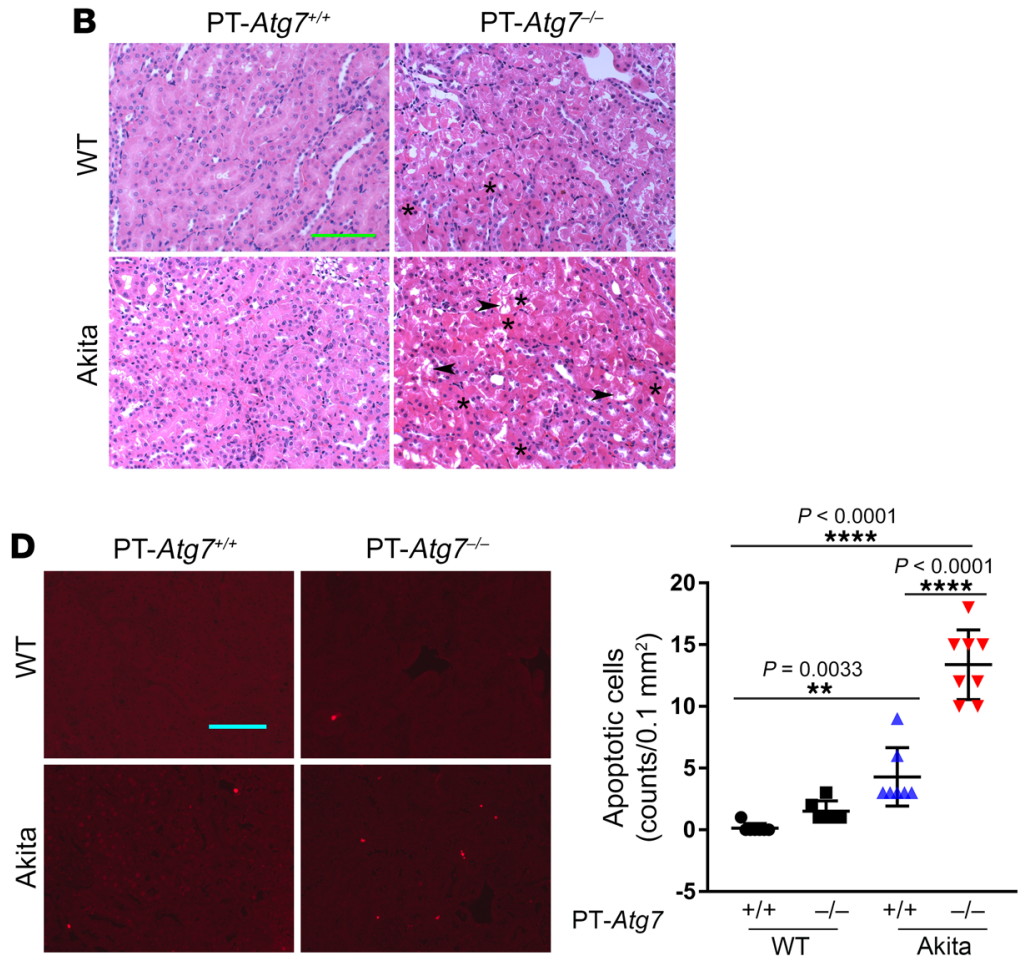

Figure 3. Autophagy deficiency in proximal tubules exaggerates renal hypertrophy and tissue damage in diabetic mice. (A) Mice of the indicated genotypes were analyzed at 14 weeks of age. PT-Atg7/-- Akita mice had a significantly higher kidney weight/body weight (KW/BW) ratio than did PT-Atg7/+ Akita mice ( $n=8-12$; 1-way ANOVA with Tukey's multiple comparisons test). (B) H\&E staining of renal histology showing more severe tubular injury in PT-Atg $7^{-1-}$ Akita mice than in the other mouse groups. Asterisks indicate acidophilic cells; arrowheads indicate lysed or severely damaged tubules. Scale bar: $0.1 \mathrm{~mm}$. (C) IHC staining of kidney cortex tissue showing macrophage infiltration in PT-Atg ${ }^{-/-}$Akita mice. Scale bar: $0.1 \mathrm{~mm}$. (D) TUNEL assay of renal cortical and outer medulla tissue showing a greater number of apoptotic cells in kidneys of PT-Atg7 ${ }^{-1-}$ Akita mice ( $n=5$-8; 1-way ANOVA with Tukey's multiple comparisons test). Scale bar: $0.1 \mathrm{~mm}$. ${ }^{*} P<0.05,{ }^{* *} P<0.01$, and ${ }^{* * * *} P<0.0001$.

examined renal cell death using TUNEL staining. We detected no TUNEL-positive cells in PT-Atg $7^{+/+}$WT kidneys. A few TUNEL-positive apoptotic cells were observed in PT-Atg $7^{-/-}$WT and PT-Atg $7^{+/+}$Akita kidneys, but significantly more were found in PT-Atg7 $7^{--}$Akita kidneys (Figure 3D). Consistently, tubular cells with active caspase-3 were detected in diabetic PT-Atg7-/- kidneys, but very few or no such cells were found in nondiabetic mice or in diabetic PT-Atg $7^{+/+}$mice (data not shown).

To verify the effects observed in Akita mice, we treated the PT-Atg7 mice with STZ to induce diabetes. Autophagy deficiency in proximal tubules did not affect the development of diabetes or body weight after STZ treatment (Supplemental Table 1). However, compared with PT- $A \operatorname{tg} 7^{+/+}$mice, PT- $A \operatorname{tg} 7^{-/-}$mice had larger kidneys and higher kidney weight/body weight ratios (Supplemental Figure 3, A and B), as well as more damaged and apoptotic tubular cells (Supplemental Figure 3, C and D) eleven weeks after STZ treatment, indicating more severe renal hypertrophy and kidney injury in PT-Atg $7^{-/-}$mice during STZ-induced diabetes.

We further monitored the long-term effects of tubular autophagy deficiency on DKD by comparing 1-year-old PT-Atg $7^{+/+}$Akita mice with PT-Atg $7^{-/}$Akita mice. With periodic acid Schiff (PAS) staining, PT-Atg $7^{-/-}$Akita mice showed more severe tubular damage than did PT-Atg $7^{+/+}$Akita mice, including loss of brush border, dilation of the tubular lumen, focal atrophy of renal tubules, and vacuolization in tubular cells (Figure 4A). Additionally, we observed significant widening of the interstitial space in the kidneys of PT-Atg7-/- Akita mice (Figure 4A). Consistently, Masson's trichrome staining detected marginal collagen deposition in renal interstitium in PT-Atg $7^{-/-}$WT and PT- $A t g 7^{+/+}$Akita mice, which was markedly increased in PT-Atg $7^{-1-}$ Akita mice (Figure 4B). PT-Atg $7^{-1-}$ Akita mice also had more renal interstitial fibronectin staining than did the other groups of mice (Figure 4C).

Functionally, both PT-Atg $7^{7^{++}}$and PT-Atg $7^{-/-}$Akita mice had a higher urine albumin/creatinine ratio (ACR) compared with WT mice, but the ACR in PT-Atg $7^{-/-}$Akita mice was significantly higher than that in PT-Atg $7^{+/+}$Akita mice (Figure 4D), suggesting that tubular autophagy deficiency exacerbates renal functional decline in diabetes. Together, these results show that autophagy defects in kidney proximal tubules may exaggerate renal hypertrophy and tissue injury in diabetes, facilitating the progression of DKD.

High-glucose incubation suppresses autophagy and induces cellular hypertrophy in renal proximal tubule cells. In vitro, high-glucose (HG) incubation induced cellular hypertrophy in rat renal proximal tubule cells (RPTCs). As shown in Supplemental Figure 4A, a forward light scatter assay showed a rightward shift in HG-treated RPTCs when compared with the cells in mannitol media for 48 hours, indicating the increased cell size during HG incubation. Quantification indicated a 7\% increase in cell size with HG incubation (Supplemental Figure 4B). Interestingly, culturing of RPTCs in normal glucose (NG) or mannitol media for 24-48 hours induced 
A

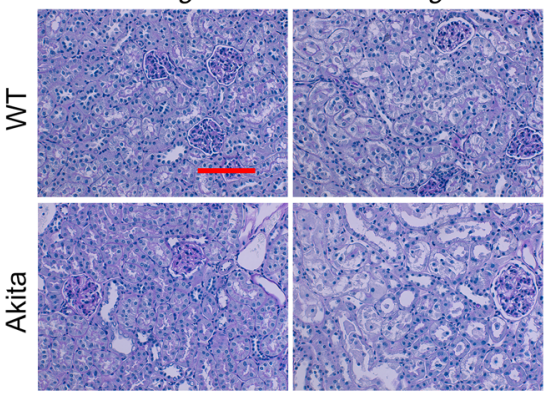

C

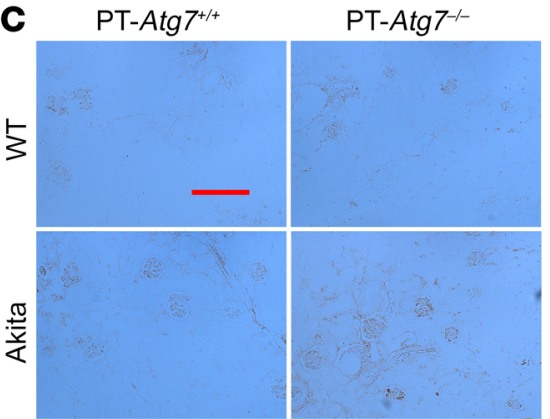

B
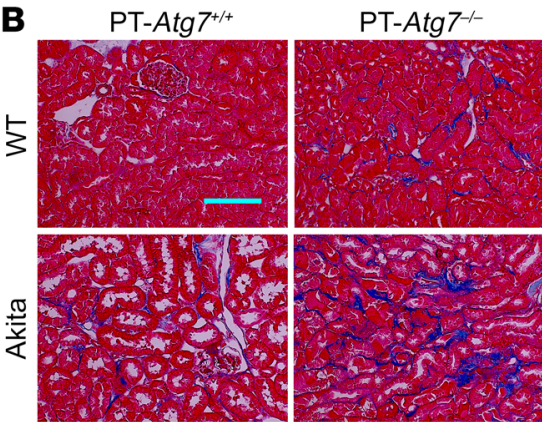

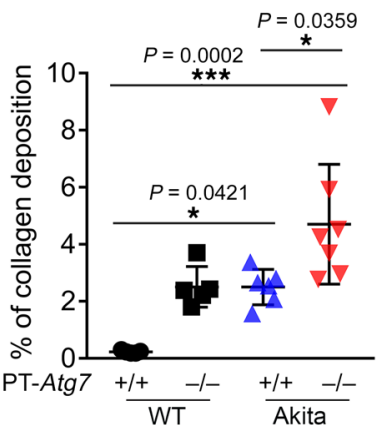

D

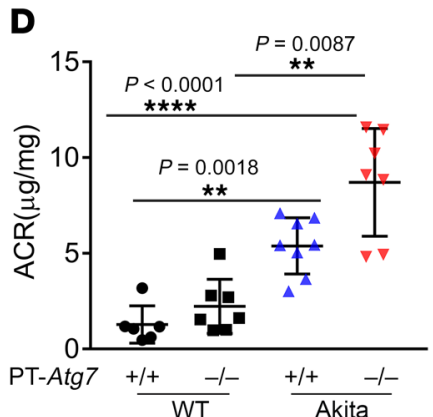

Figure 4. Long-term effects of tubular autophagy deficiency on diabetic kidneys shown in 1-year-old mice. (A) PAS staining showing worse tubular damage in PT-Atg 7---Akita mice. Scale bar: $0.1 \mathrm{~mm}$. (B) Masson's trichrome staining showing more collagen deposition in renal interstitium in PT-Atg7-/- Akita mice ( $n=5-8$; 1-way ANOVA with Tukey's multiple comparisons test). Scale bar: $0.1 \mathrm{~mm}$. (C) IHC staining showing more interstitial fibronectin in PT-Atg7-/Akita mice ( $n=5$; 1-way ANOVA with Tukey's multiple comparisons test). Scale bar: $0.1 \mathrm{~mm}$. (D) ACR analysis showing the highest ACR in PT-Atg7-1- Akita mice ( $n=6-8$; 1-way ANOVA with Tukey's multiple comparisons test). ${ }^{*} P<0.05,{ }^{* *} P<0.01,{ }^{* *} P<0.001$, and ${ }^{* * *} P<0.0001$.

the autophagy marker LC3-II, which was abolished in HG-incubated cells, indicating defective autophagy (Supplemental Figure 4C). To further clarify this defect, we analyzed autophagic flux by testing the effect of chloroquine, an autolysosomal inhibitor. As shown in Supplemental Figure 4D, chloroquine increased LC3-II levels in both NG- or mannitol-treated and HG-treated RPTCs, but LC3-II levels in the HG-treated cells were still significantly lower than those in the NG-treated cells, suggesting that there was a problem with autophagy initiation in the HG-treated cells. In cells transfected with GFP-LC3, the formation of GFP-LC3labeled autophagic puncta was also significantly decreased by HG incubation (Supplemental Figure 5A). HG similarly suppressed autophagy in the human HK2 proximal tubule cell line (Supplemental Figure 5B). We next investigated the effect of autophagy inhibition on cellular hypertrophy. As shown in Supplemental Figure 5C, autophagy inhibition by chloroquine induced a rightward shift in a forward light scatter assay, indicating an increase in cell size in RPTCs. Chloroquine increased cell size in HK2 cells as well (Supplemental Figure 5, D and E). Together, these cell culture studies indicate that HG incubation may suppress autophagy initiation to promote renal tubule cell hypertrophy.

Downregulation of ULK1 in diabetic kidneys and HG-incubated renal tubule cells. To understand the mechanism of autophagy impairment in diabetic kidneys, we first analyzed key autophagy proteins in Akita mice by immunoblotting. We found that Akita mice and their WT littermates expressed similar levels of Beclin1 and the Atg5-Atg12 complex in kidneys, whereas Akita mice expressed significantly less ULK1 and Atg7 at both 7 and 14 weeks of age (Figure 5A and Supplemental Figure 6A). IHC staining further confirmed a decrease in ULK1 in diabetic kidneys, especially in renal tubules, and, notably, although some tubules lost ULK1 staining, others maintained it (Figure 5B). Similarly, renal expression of ULK1 was reduced in STZ-induced diabetic mice (Figure 5, C and D). However, STZ treatment did not change Atg7, Atg5, or Beclin1 expression in mouse kidneys (Figure 5C). These results led us to focus on ULK1, a key component in the autophagy initiation complex $(12,32)$. Consistent with the in vivo observations, HG incubation decreased ULK1 expression in RPTCs at 9, 24, and 48 hours (Supplemental Figure 6B). HG also reduced the phosphorylation of ULK1 at Ser555 and Ser757, two critical regulatory sites (ref. 33 and Supplemental Figure 6C). Thus, both in vivo and in vitro experiments demonstrated that a prominent molecular change in diabetic kidneys was the downregulation of ULK1, which may mediate autophagy impairment in diabetes.

miR-214 is induced in diabetic kidneys to repress ULK1 for autophagy impairment, renal hypertrophy, and DKD. To delineate the mechanism of ULK1 downregulation in diabetic kidneys, we first measured its mRNA levels and showed that HG incubation did not reduce ULK1 mRNA levels in RPTCs (Supplemental Figure 7A). Akita diabetic mice did not show a decrease in ULK1 mRNA levels in kidneys either (Supplemental Figure 7B). In STZ-induced diabetic mice, ULK1 mRNA levels did not decrease in kidneys at 5 weeks and decreased marginally (13\%) at 11 weeks (Supplemental Figure 7C), whereas ULK1 protein expression decreased by approximately $60 \%$. These results indicate that ULK1 downregulation in diabetic kidneys may mainly occur at the posttranscriptional level. 
A

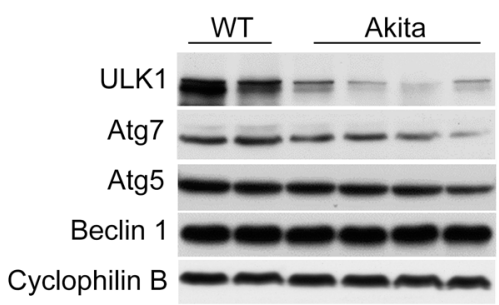

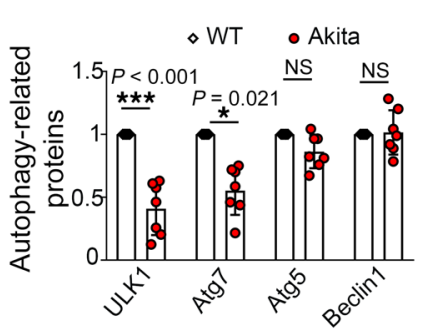

B

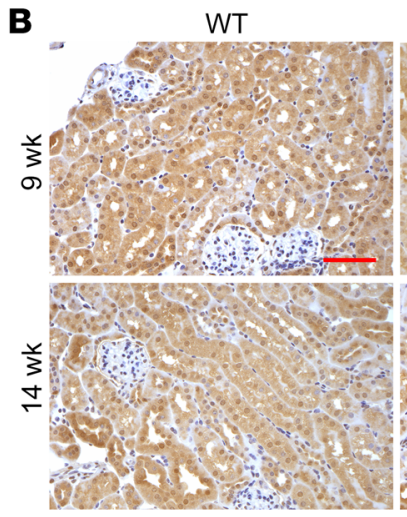

Akita

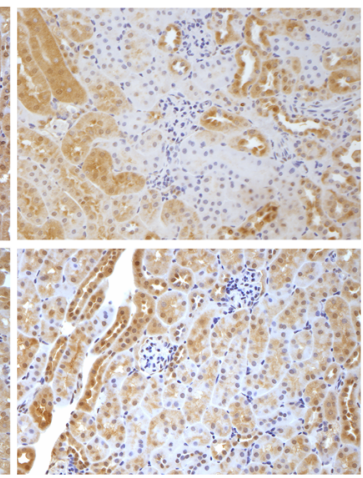

C

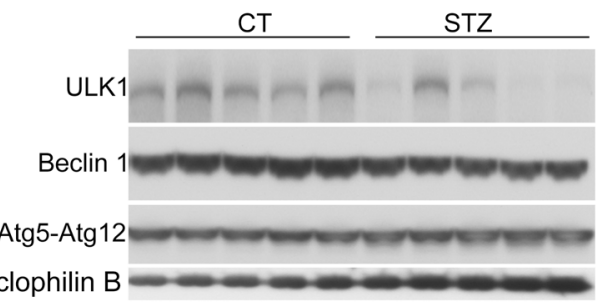

Cyclophilin B

$\operatorname{Atg} 7$

$\mathrm{CT}$

Cyclophilin B
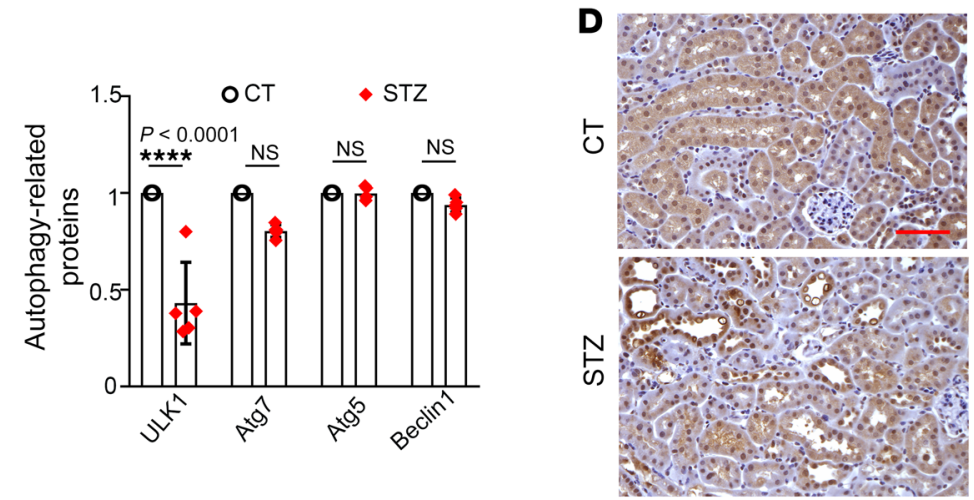

Figure 5. Downregulation of ULK1 in kidney tubules in diabetes. (A) Immunoblots and densitometric analysis showing decreased levels of ULK1 and Atg7 in kidney cortex tissues from 7- to 9-week-old Akita mice compared with levels in WT controls ( $n \geq 6$; 2 -tailed Student's $t$ test). (B) IHC staining showing reduced expression of ULK1 in kidney tissues from 9- to 14-week-old Akita mice. Scale bar: $0.1 \mathrm{~mm}$. (C) Immunoblots and densitometric analysis showing decreased ULK1 expression in kidney cortex samples from STZ-treated C57BL/6 mice ( $n \geq 5 ; 2$-tailed Student's $t$ test). Control mice were treated with vehicle (citrate) solution. (D) IHC staining of ULK1 showing reduced expression of ULK1 in kidney tubules from STZ-treated mice. Scale bar: $0.1 \mathrm{~mm}$. ${ }^{*} P<0.05$, ${ }^{* *} P<0.001$, and ${ }^{* * *} P<0.0001$.

Fip200 and Atg13, two proteins of the autophagy initiation complex, may regulate the stability of ULK1 (34-36). However, HG incubation of RPTCs did not change the expression levels of Fip200 or Atg13 (Supplemental Figure 7D). Furthermore, the proteasome inhibitor MG132 could not prevent the decrease in ULK1 expression during $\mathrm{HG}$ incubation, negating a role of proteasomal degradation (Supplemental Figure 7E). In addition, mTOR-mediated phosphorylation of AMBRA1 at Ser52 may induce its interaction with the E3 ligase TRAF6, resulting in ULK1 degradation (37). In HG-treated RPTCs, we observed no increase in phosphorylated AMBRA1 (p-AMBRA1) (Ser52), despite mTOR activation shown by phosphorylation of P70S6K, suggesting that the mTOR/ AMBRA1 pathway is not responsible for decreased ULK1 expression in diabetes (Supplemental Figure 7F).

With these negative results, we hypothesized that a decrease in ULK1 in diabetes may involve specific microRNAs, which repress target gene expression posttranscriptionally by inducing mRNA degradation or preventing their translation into proteins (38). To identify the specific microRNAs, we first used the miRanda microRNA target prediction database (http://www.microrna. org) to predict the potential microRNAs that may target ULK1. We then concentrated on those microRNAs that had been implicated in diabetes and kidney diseases. The bioinformatics analysis indicated miR-214 as a potential microRNA that may target ULK1 in diabetic kidneys. We identified a conserved miR-214 targeting site in the $3^{\prime}$-UTR of the ULK1 gene in various animal species ranging from Xenopus tropicalis to humans (Figure 6A). We further detected a gradual increase in miR-214 expression in kidneys in both Akita mice and STZ-induced diabetic mice (Figure 6, B and C). miR214 expression in the kidneys of Akita mice was approximately 1.4-fold over that in WT mouse kidneys 3 weeks after the onset of diabetes, which increased to approximately 1.8 -fold by 10 weeks. Similarly, STZ-treated C57BL/6 mice had approximately 1.3- and 1.9-fold increases in miR-214 expression 5 and 11 weeks after diabetes induction, respectively. Using ISH, we further localized miR-214 induction in diabetic kidneys predominantly in proximal tubules but not in glomeruli (Figure 6D and Supplemental Figure $8 \mathrm{~A}$ ), and the number of miR-214-positive tubular cells increased markedly in Akita diabetic kidneys as compared with nondiabetic WT tissues. In vitro in RPTCs, HG induced a $30 \%$ increase in miR214 (Figure 6E). Together, these results demonstrate the induction of miR-214 in kidney tubule cells during diabetes, accompanied by the downregulation of ULK1.

To determine whether ULK1 is a real target of miR-214, we first transfected miR-214 into human embryonic kidney 293 (HEK293) cells, which led to a significant decrease in ULK1 expression (Figure 7A and Supplemental Figure 8B). Conversely, inhibition of miR-214 with anti-miR-214 locked nucleic acid (LNA) increased 
A

3' $^{\prime}$ ugacggacagacacgGACGACa 5' hsa-miR-214
5' ggaccucaccagggaCUGCUGg 3' ULK1 3'UTR (503-524 bp)

miR-214 target sequence
D $\quad \operatorname{miR}-214$
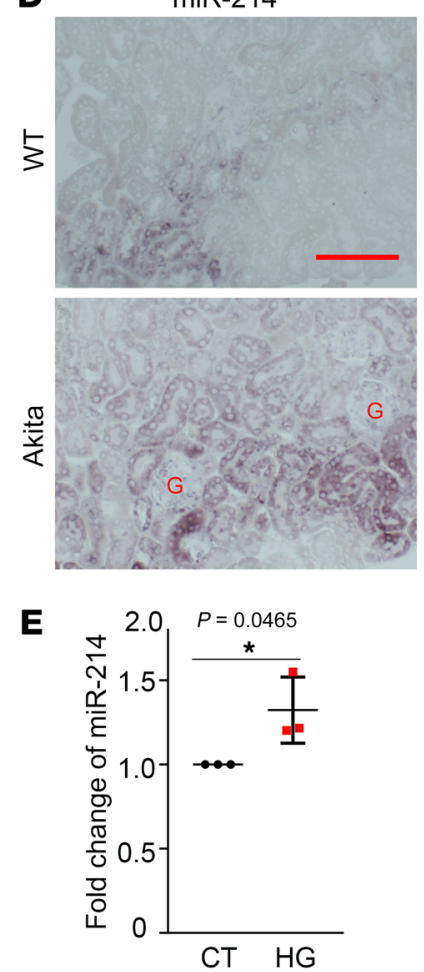

Figure 6. miR-214 is induced in diabetic kidneys and HG-treated tubular cells. (A) Conserved miR-214 target sequence in the 3'-UTR of the ULK1 gene in various animal species. (B) Real-time PCR analysis showed that expression of miR-214 was higher in kidney cortex of Akita mice with 3 and 10 weeks of diabetes than in that of nondiabetic WT mice, ( $n=6$; 2-tailed Student's $t$ test). (C) miR-214 expression was higher in kidneys of STZ-treated C57BL/6 mice at 5 or 11 weeks of age than in kidneys of control mice, as shown by real-time PCR ( $n=4$ or 6 ; 2-tailed Student's $t$ test). (D) ISH showing higher miR-214 expression in kidney tubules of 14-week-old Akita diabetic mice than nondiabetic WT mice. G, glomeruli. Scale bar: $0.1 \mathrm{~mm}$. (E) Expression of miR-214 was higher in HG-treated RPTCs at 5 hours than in control cells, as shown by real-time PCR ( $n=3 ; 2$-tailed Student's $t$ test). ${ }^{*} P<0.05,{ }^{* *} P<0.01$, and ${ }^{* * * *} P<0.0001$.

ULK1 expression (Figure 7B, lane 2 vs. lane1). Moreover, anti-miR214 prevented an HG-induced decrease in the expression of ULK1 (Figure 7B, lane 4 vs. lane 3) and LC3-II (Figure 7C, lane 4 vs. lane 3), further suggesting a role of miR-214 in ULK1 downregulation and autophagy impairment under diabetic conditions. We further sought to determine whether ULK1 is a direct target of miR-214 by performing a microRNA target luciferase reporter assay. The 3'-UTR of ULK1 was placed downstream of the luciferase reporter gene driven by a constitutive promoter. The construct was transfected into RPTCs along with miR-214-expressing plasmids or empty vectors. As shown in Figure 7D, cotransfection of miR-214 reduced luciferase expression in luciferase-ULK1 3'-UTR-transfected cells. Notably, HG incubation also suppressed luciferase expression in transfected cells, and this effect was partially diminished when the targeting sequence of miR-214 was mutated in the luciferase-ULK1 3'-UTR (Figure 7E). Collectively, these data indicate that ULK1 is a direct target of miR-214 and that miR-214 may repress ULK1 expression in diabetes.

To examine miR-214 regulation of ULK1 in vivo, we tested the effects of anti-miR-214 LNA in Akita mice. Anti-miR-214, but not the scrambled sequence, suppressed the increase in miR-214 in Akita mice (Supplemental Figure 8C). Importantly, anti-miR-214 partially prevented the decrease in ULK1 in Akita mouse kidneys, whereas the scrambled sequence LNA had no effect (Supplemental Figure 8D). Anti-miR-214 also partially preserved kidney tubular autophagy in Akita diabetic mice as shown by LC3 puncta (Supplemental Figure 8E). Moreover, compared with scrambled LNA, anti-miR-214 reduced the kidney weight/body weight ratio in Akita mice (Supplemental Figure 8F), supporting a role of miR214 in renal hypertrophy in diabetes.

To confirm the results of anti-miR-214 and further elucidate the pathogenic role of miR-214 in diabetic kidneys, we established a proximal tubule-specific miR-214-knockout (PT-miR-214-/-) mouse model by breeding miR-214-floxed mice (39) with PEPCKCre mice (40). PT-miR-214/- mice were further bred with Akita mice to generate PT- $m i R-214^{+/+}$and PT- $m i R-214^{-/-}$Akita mice, which were then compared to determine the effects of proximal tubule miR-214 ablation on diabetic kidneys (Supplemental Figure 9). Compared with nondiabetic WT mice, both PT-miR-214-/-- and PT-miR-214 ${ }^{+/+}$Akita mice had high levels of fasting blood glucose and low body weights at 6,10 , and 20 weeks of age (Supplemental Table 2). PT-miR-214 ${ }^{+/+}$Akita mice showed an induction of miR-214 in kidneys, which was diminished in PT-miR-214 ${ }^{--}$Akita mice (Supplemental Figure 10A). Remarkably, PT- $m i R-214^{+/+}$Akita mice showed decreased expression of ULK1 and LC3-II in kidneys, which, for both proteins, was partially prevented in PT-miR-214 ${ }^{-/}$ Akita mice, supporting a role of miR-214 in ULK1 and autophagy regulation in diabetic kidneys (Figure 7, F and G). Consistently, PT- $m i R-214^{-/}$Akita mice appeared to have more LC3 puncta in kidney tubules than did PT-miR-214 ${ }^{+/+}$Akita mice (Supplemental Fig- 
A

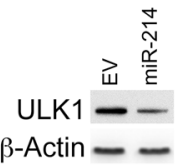

$$
\text { B }
$$
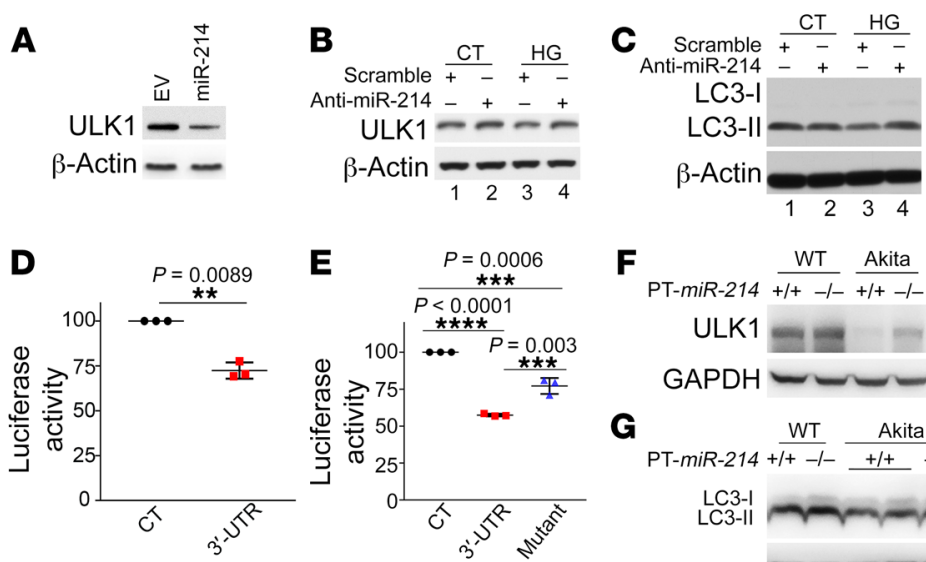

$\mathbf{F}$

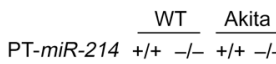

ULK1

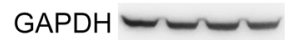

G
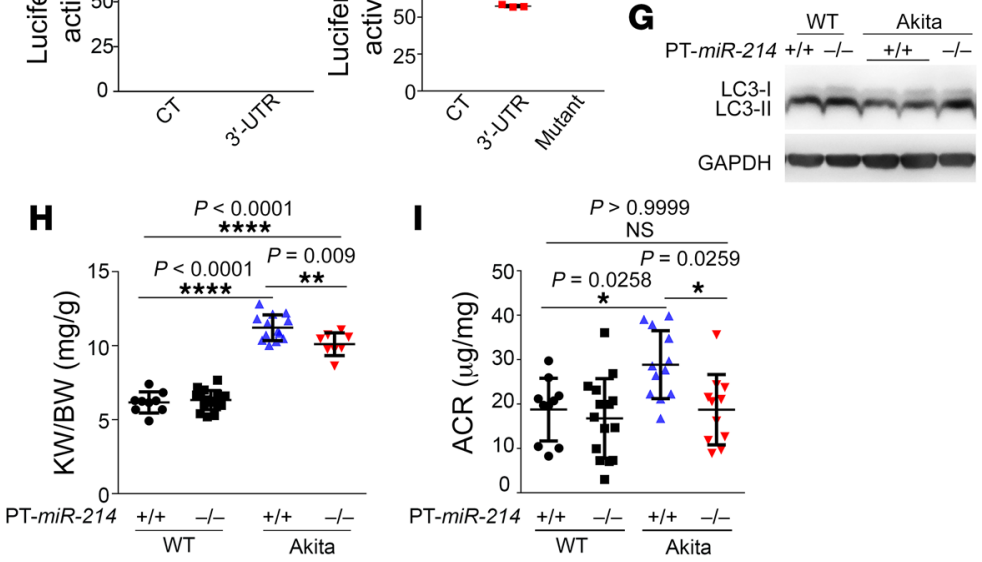

Figure 7. Upregulated miR-214 represses ULK1 for autophagy impairment, renal hypertrophy, and DKD. (A) Immunoblot shows a miR-214-induced decrease in ULK1 expression. HEK293 cells were transfected with miR-214 plasmids or empty vectors (EV) for 24 hours to collect whole-cell lysate for immunoblot analysis. (B and C) RPTCs were transfected with a scrambled sequence or anti-miR-214 LNA and then incubated with NG (CT) or $30 \mathrm{mM} \mathrm{HG}$ media for 24 hours to collect whole-cell lysate for immunoblot analysis. HG incubation induced decreases in ULK1 (B) and LC3 (C) expression, which were prevented by anti-miR-214. (D) RPTCs were cotransfected with luciferase plasmids or luciferase-ULK1 3'-UTR plasmids together with miR-214 plasmids for 24 hours. Cell lysates were then collected to determine luciferase activity. miR-214 decreased luciferase activity in luciferase-ULK1 3'-UTR-transfected cells ( $n=3$; 2-tailed Student's $t$ test). (E) RPTCs were cotransfected with luciferase, luciferase-ULK1 3'-UTR, or luciferase ULK1 $3^{\prime}$-UTR-mutant plasmids to establish 3 stable cell lines, which were then treated with $30 \mathrm{mM} \mathrm{HG}$ for 24 hours. Cell lysates were collected to determine luciferase activity. HG treatment decreased luciferase activity in luciferase-ULK1 3'-UTRtransfected cells, but there was less effect in the cells transfected with luciferase ULK1 3'-UTR mutant ( $n=3$; 1-way ANOVA with Tukey's multiple comparisons test). (F) ULK1 was downregulated in the kidneys of PT-miR-214+/+ Akita mice, an effect that was partially prevented in PT-miR-214-/- Akita mice. (C) Expression of LC3-II was decreased in the kidneys of PT-miR-214+/+ Akita mice and was suppressed in PT-miR-214/- Akita mice. (H) PT-miR-214+/+ Akita mice showed a higher kidney weight/body weight ratio than did WT mice, which was reduced in PT-miR-214-/- Akita mice ( $n \geq 8$; 1-way ANOVA with Tukey's multiple comparisons test). (I) Compared with WT mice, PT-miR-214+/+ Akita mice had a higher ACR, and PT-miR-214/- Akita mice had a markedly reduced ACR ( $n \geq 9$; 1-way ANOVA with Tukey's multiple comparisons test. ${ }^{*} P<0.05$, ${ }^{* *} P<0.01$, ${ }^{* * *} P<0.001$, and ${ }^{* * *} P<0.0001$.

ure 10B). Moreover, PT-miR-214 ${ }^{-/}$Akita mice had smaller kidneys and kidney weight/body weight ratios than did PT-miR-214 ${ }^{+/+} \mathrm{Aki}-$ ta mice, suggesting that miR-214 contributes to renal hypertrophy in diabetes (Supplemental Figure 10C and Figure 7H). Remarkably, PT-miR-214 $/$ Akita mice also had significantly better renal function, as indicated by a much lower urine ACR than was detected in PT-miR-214 ${ }^{+/+}$Akita mice (Figure 7I).

Collectively, these pharmacological and gene-knockout studies indicate that miR-214 is induced in diabetes to repress ULK1 in kid- ney tubule cells, leading to autophagy impairment, tubular hypertrophy, and a decline of renal function in DKD.

P53 mediates miR-214 induction in diabetic kidneys for autophagy impairment, renal hypertrophy, and DKD. How is miR-214 induced in DKD? With this question in mind, we screened several possible upstream transcription factors, among which p53 was markedly upregulated and activated in the kidneys of both Akita and STZ-treated mice (Figure 8A and Supplemental Figure 11A). In vitro, HG incubation of RPTCs also induced p53 activation (phosphorylation at Ser15) (Figure 8B). Pifithrin- $\alpha$, a pharmacological inhibitor of p53, prevented the decreases in ULK1 and LC3-II expression in HG-treated RPTCs (Figure 8, C and D: lane 4 vs. lane 2). Notably, pifithrin- $\alpha$ also decreased miR-214 induction in HG-treated cells (Figure 8E). These results suggest that p53 may mediate miR-214 induction in kidney tubule cells to suppress ULK1 and autophagy in diabetes.

We then analyzed the transcription factor binding profile of the miR-214 gene using the JASPAR database (http://jaspar.genereg.net/) and identified a putative p53 binding site in its promoter region (Figure $8 \mathrm{~F}$ ). To verify p53 binding to this site, we performed a ChIP assay to detect the binding site sequence in anti-p53 immunoprecipitation. As shown in Figure 8G, HG incubation induced a 2.3-fold increase in $\mathrm{p} 53$ binding to the miR-214 gene promoter sequence with the putative binding site. To clarify whether $\mathrm{p} 53$ regulates miR-214 in vivo, we used the proximal tubule-specific p53-knockout (PT-p53--) mouse model from our previous work (41). PT- $p 53^{-/-}$mice and their PT- $p 53^{+/+}$littermates were treated with STZ

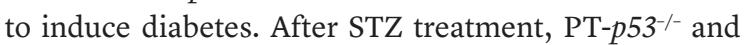
PT- $p 53^{+/+}$mice had similar increases in blood glucose and loss of body weight (Supplemental Table 3), indicating the development of diabetes. However, PT- $p 53^{+/+}$ mice showed a marked ( $>2$-fold) induction of miR-214 in kidneys after STZ treatment, which was attenuat-

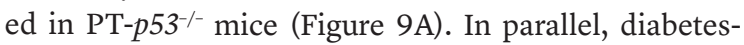
associated decreases in ULK1 and LC3-II expression were

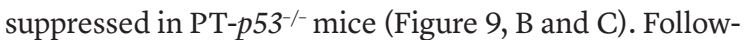
ing STZ induction of diabetes, PT- $p 53^{-/}$kidneys also had more LC3-positive puncta or autophagosomes in renal tubules than did PT-p53 $3^{+/}$kidneys (Figure 9D). In PAS staining, diabetic PT- $p 53^{+/+}$mice showed obvious renal histopathological changes, including tubular dilation, loss of brush border, and tubular atrophy, which were substantially decreased in diabetic PT-p53/- mice (Figure 9E). Kidney weight and the kidney weight/body weight ratio increased during diabetes, but the increase was partially suppressed in $\mathrm{PT}-\mathrm{p} 53^{-/-}$mice, suggesting less renal hypertrophy in these animals (Supplemental Figure 11B and Figure 9F). Remarkably, PT- $p 53^{--}$mice had a significantly lower ACR than did PT- $p 53^{+/+}$mice in STZ-induced diabetes (Figure 9G), indicating better renal function. Together, these results suggest that p53 is activated in diabetes to induce miR-214, which then represses ULK1 expression, resulting in autophagy impairment, renal hypertrophy, and DKD. 
A

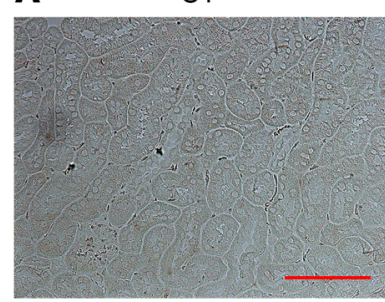

D

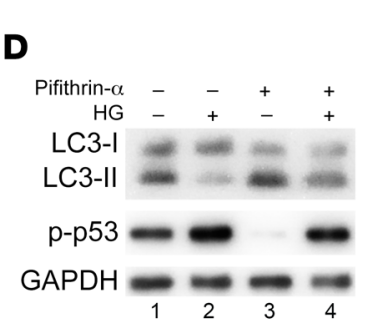

STZ

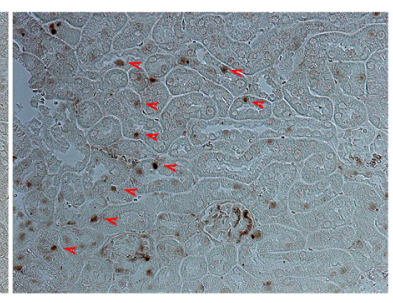

B

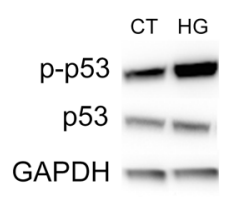

C

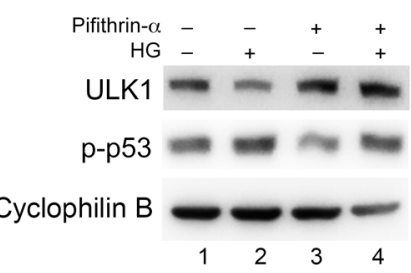

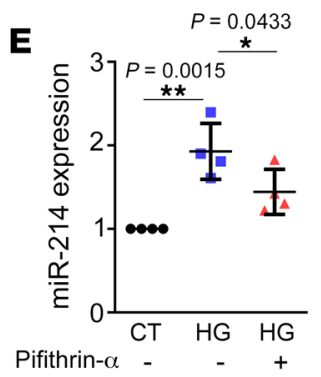

$\mathbf{F}$
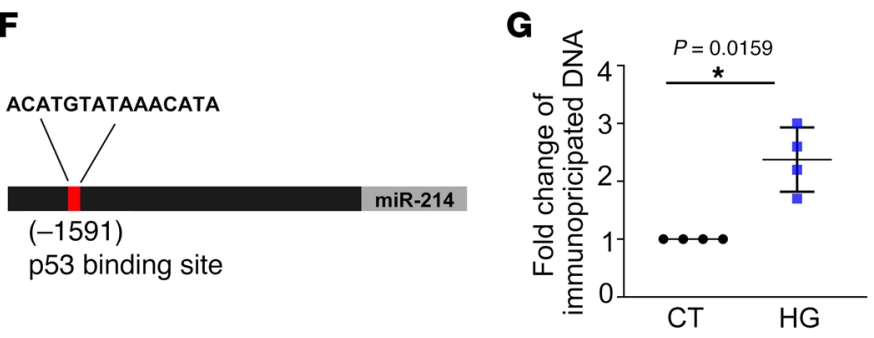

Figure 8. p53 is activated in diabetic kidneys to induce miR-214 for ULK1 and autophagy suppression. (A) p53 induction in kidney tubule cells of STZ-treated diabetic mice shown by IHC staining. C57BL/6 mice were treated with STZ or control vehicle solution and examined 11 weeks later for p53 IHC. Arrowheads indicate p53-positive nuclei. Scale bar: $0.1 \mathrm{~mm}$. (B) Immunoblots showing p53 and its phosphorylation induced by HG in RPTCs. RPTCs were incubated with $5.5 \mathrm{mM} \mathrm{NG} \mathrm{(CT)} \mathrm{or} 30 \mathrm{mM} \mathrm{HC}$ for 24 hours to collect lysate for immunoblot analysis. (C and D) Inhibition of ULK1 and LC3 decreases in HG-treated RPTCs by pifithrin- $\alpha$. RPTCs were incubated with NG or $30 \mathrm{mM} \mathrm{HG}$ in the absence or presence of $5 \mu \mathrm{M}$ pifithrin- $\alpha$ for 24 hours to collect wholecell lysate for immunoblot analysis. (E) Inhibitory effect of pifithrin- $\alpha$ on miR-214 induction during HC incubation shown by real-time PCR analysis ( $n=4$; 1-way ANOVA with Tukey's multiple comparisons test). (F) miR-214 promoter region harboring the p53 binding site. (G) ChIP assay of p53 binding to the miR-214 promoter sequence during HG incubation. BUMPT cells were treated with $5.5 \mathrm{mM}$ (control) or $30 \mathrm{mM}$ (HG) glucose for 24 hours for the ChIP assay using anti-p53 antibody ( $n=4$; 2-tailed Student's $t$ test). The values were normalized to that for the control group, which was arbitrarily set at 1 . ${ }^{*} P<0.05$.

Correlations between p53, miR-214, ULK1, and LC3 in renal biopsies from patients with diabetes. Our results from animal and cell models unveiled the p53/miR-214/ULK1 pathway that leads to defective autophagy, renal hypertrophy, and a decline in renal function in diabetes. To determine the clinical relevance of the findings, we examined the expression of p53, miR-214, ULK1, and LC3 in renal biopsies from patients with diabetes. The patients' characteristics are presented in Supplemental Table 4. Nondiabetic samples were obtained from normal peritumoral kidney tissues. In IHC analysis, none of the control renal biopsies had positive p53 staining, whereas 15 of 20 renal biopsies from patients with diabetes showed p53 staining (Figure 10A). Notably, in the diabetic kidney samples, p53 was predominantly expressed in the cell nuclei of dilated renal tubules. ISH revealed that only 2 of 14 control renal biopsies had positive miR-214 staining compared with positive staining in 15 of 20 renal biopsies from patients with diabetes (Figure 10B). Interestingly, miR-214 was detected in dilated kidney tubules as p53, but mainly in the cytoplasm. On the contrary, the majority of renal tubules in control renal biopsies stained strongly for ULK1, whereas many tubules in diabetic renal biopsies had low ULK1 staining (Figure 11A). Compared with control biopsies, the renal biopsies from patients with diabetes also showed obviously fewer LC3 puncta or autophagosomes (Figure 11B). Furthermore, linear regression analysis showed a significant positive correlation between p53 and miR-214 expression (Figure 12A) and between ULK1 and LC3 expression (Figure 12B) in diabetic renal biopsies. In contrast, we noted a significant negative correlation between expression of miR-214 and ULK1 (Figure 12C), and between expression of $\mathrm{p} 53$ and ULK1 (Figure 12D). These results provide further support for a p53/miR-214/ULK1 axis in autophagy impairment in diabetes and establish its clinical relevance.

We further analyzed the correlation between these autophagy impairment-related molecular changes and clinical parameters in patients with diabetes and detected no correlations between these molecular changes and the glomerular filtration rate (GFR) or proteinuria. We then analyzed the correlation of these molecular changes with clinical pathologies, which include interstitial fibrosis and tubular atrophy (IFTA), interstitial inflammation, and vascular lesions, according to the guidelines for pathological classification of diabetic nephropathy (42). We found no correlation between $\mathrm{p} 53$ and IFTA $(P=0.1732)$, or between miR-214 and IFTA $(P=0.0721)$. However, we noted negative correlations between ULK1 expression and IFTA $(Y=-0.1063 X+2.337, P=0.0012)$, and between LC3 and IFTA $(Y=-0.1353 X+2.588, P=0.0058)$.

Similarly, we found no correlation between p53 or miR-214 and interstitial inflammation ( $P=0.0781$ and $P=0.0698$, respectively), but we noted negative correlations between ULK1 expression and IFTA $(Y=-0.1288 X+1.718, P=0.0012)$, and between LC3 and IFTA $(Y=-0.1174 X+1.609, P=0.0058)$. As expected, no correlation was found between p53, miR-214, ULK1, LC3 and vascular lesions, including the arteriolar hyalinosis score and arteriosclerosis.

To further verify the correlations between the molecular changes and renal fibrosis, we analyzed fibrosis in human renal biopsies by Sirius Red/Fast Green Collagen staining. In this assay, diabetic kidneys had more fibrosis than nondiabetic kidneys (Supplemental Figure 12, A and B). Linear correlation and regression analyses demonstrated significant positive correlations of $\mathrm{p} 53$ and miR-214 with renal fibrosis (Supplemental Figure 13, A and B). In 


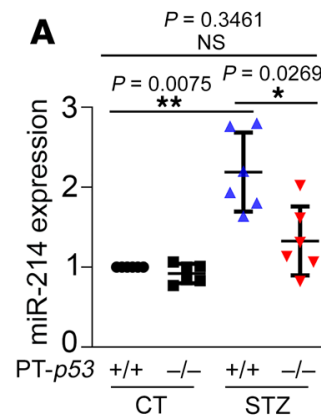

B

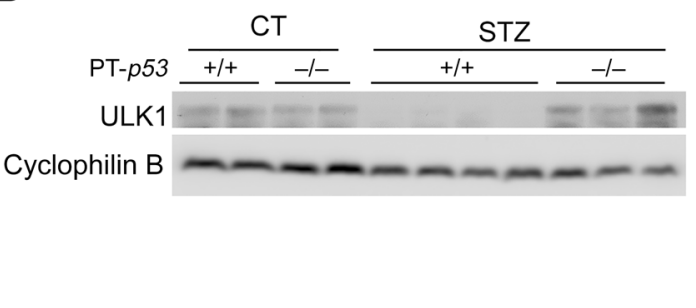

C

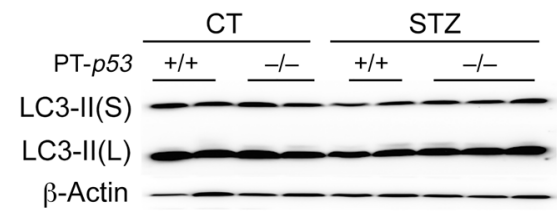

D $\quad \mathrm{PT}-p 53^{+/+}$
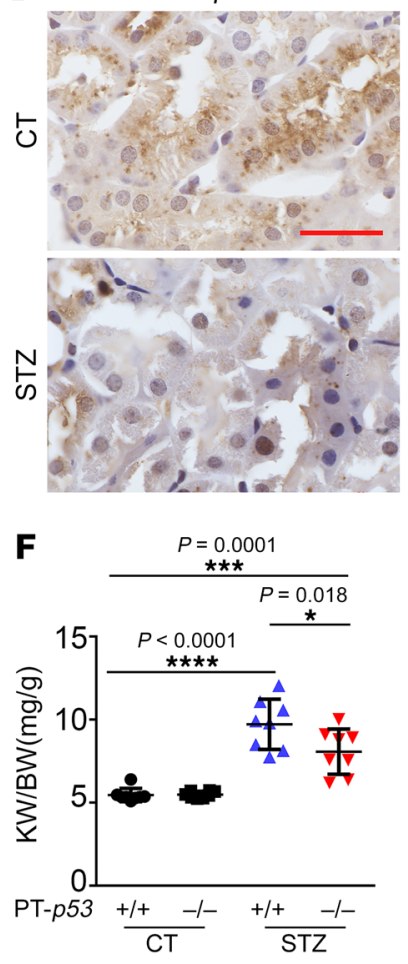

$\mathrm{PT}-\mathrm{p} 53^{--}$

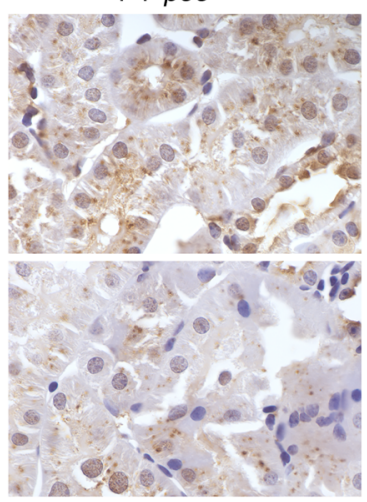

G

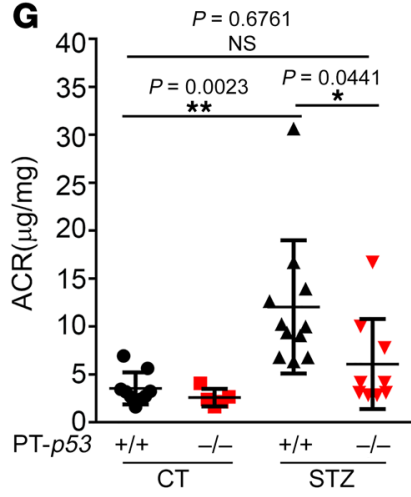

E

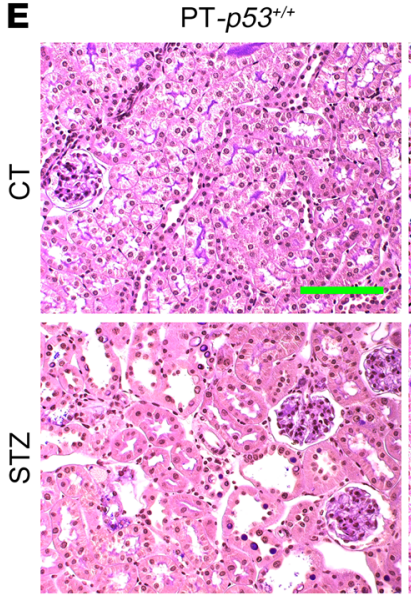

PT-p53--

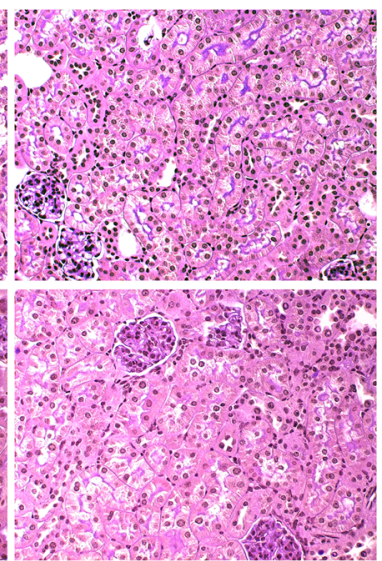

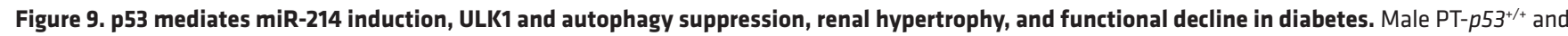

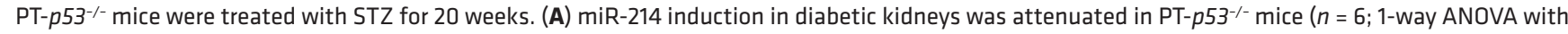

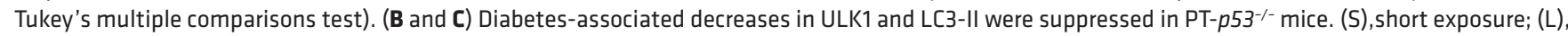
long exposure. (D) Diabetes-associated decrease in LC3 puncta in kidney tubules was partially recovered in PT-p53/- mice. Scale bars: $50 \mu \mathrm{m}$. (E) Diabe-

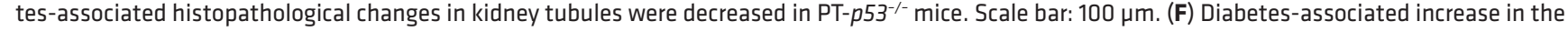

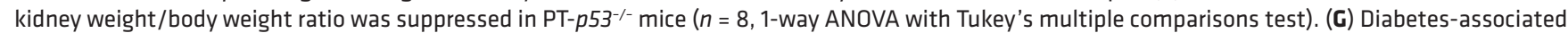
increase in ACR was diminished in PT- $p 53^{-/-}$mice ( $n=5$ for PT- $p 53^{-1-}$ WT, $n=9$ for others, 1-way ANOVA with Tukey's multiple comparisons test). ${ }^{*} P<0.05$, ${ }^{* *} P<0.01,{ }^{* *} P<0.001$, and ${ }^{* * *} P<0.0001$.

contrast, we found negative correlations between ULK1 and LC3 expression and renal fibrosis (Supplemental Figure 13, C and D). These results support a relationship between the p53/miR-214/ ULK1 axis, autophagy impairment, and tubule interstitial pathologies in DKD.

\section{Discussion}

In the current study, we show that autophagy was suppressed in both cell culture and mouse models of DKD. In addition to traditional analyses, we detected autophagy dysfunction in DKD by using RFP-GFP-LC3 autophagy reporter mice to monitor auto- phagosome formation and its maturation to an autolysosome. We further detected decreases in ULK1 and LC3 expression in diabetic patients' kidneys. These findings, together with other studies (43), demonstrate that autophagy is impaired in DKD. Functionally, the current study provides convincing evidence that autophagy impairment in kidney tubules contributes to renal hypertrophy and related pathologies leading to DKD. Especially noteworthy is our finding that autophagy deficiency in kidney proximal tubules (PT-Atg7--) exaggerated renal hypertrophy and DKD in both Akita and STZ-induced diabetic mice. Blockade of autophagy by chloroquine also induced hypertrophy in cultured renal tubule cells. 
A

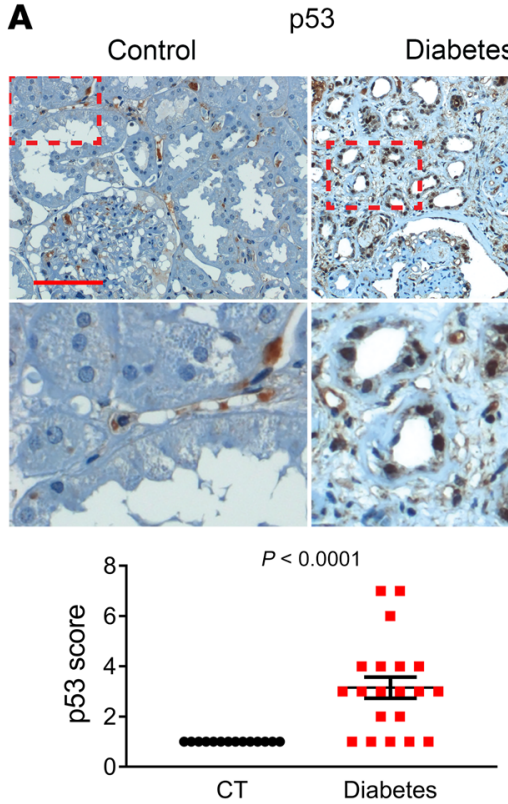

B
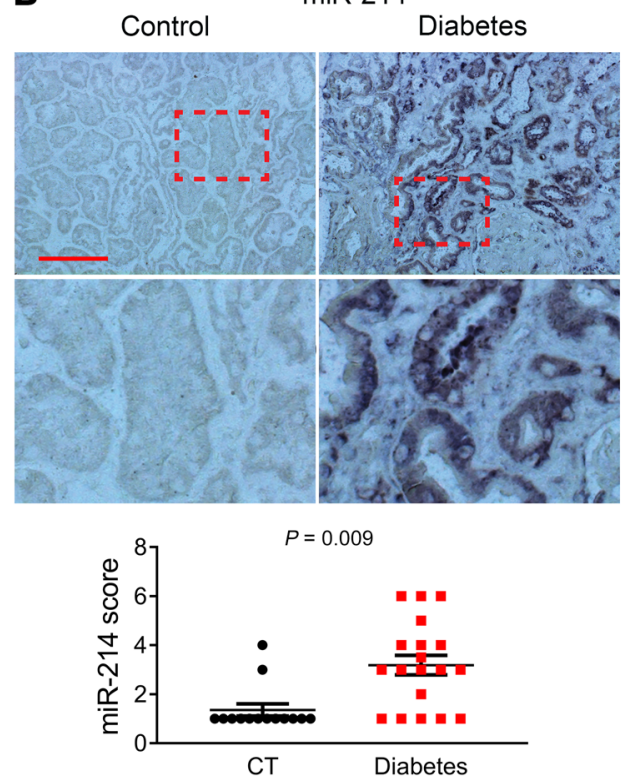

Figure 10. p53 activation and miR-214 induction in human diabetic kidneys. Renal biopsies from diabetic patients and nondiabetic patients were examined for the expression of p53 by IHC (A) and of miR-214 by ISH (B). $n=14$ control and $n=20$ diabetic renal biopsies. Scale bars: $0.1 \mathrm{~mm}$. Original magnification, $\times 200$ (insets). Compared with the control, the renal biopsies from patients with diabetes showed significantly higher expression of $\mathrm{p} 53$ and miR-214 in kidney tubules (2-tailed Student's $t$ test).
The effect of hypertrophy was not surprising, because autophagy is a catabolic pathway whose inhibition may prevent cytoplasmic degradation and increase cell size. Renal hypertrophy is an early pathological change in diabetes that contributes to the development and progression of DKD. Consistently, we found that autophagy deficiency in kidney tubules resulted in worse tubular injury, interstitial fibrosis, inflammation, and albuminuria in Akita diabetic mice (Figures 3 and 4). Similarly, tubular autophagydeficient mice showed worsened inflammation and renal fibrosis in STZ-induced diabetes (43). Collectively, these results indicate that renal tubular autophagy is protective in DKD and that its decrease is pathogenic.

Although we only examined mouse models of type 1 diabetes, the key findings were recapitulated by HG incubation of renal tubule cells (Supplemental Figures 4-6) and in renal biopsies from patients with type 2 diabetes mellitus (Figures 10-12). Together with the previous demonstration of autophagy suppression in obesity-related or type 2 diabetes, these results suggest that autophagy impairment may be a common feature of both type 1 and type 2 diabetes, thus conferring an important pathogenic mechanism in DKD and providing potential therapeutic strategies. The latest work by Sakai et al. (23) consistently demonstrated autophagy dysfunction in both STZ-induced type 1 and $d b / d b$ type 2 diabetic mice. In this study, we identified a defect at autolysosomes called lysosomal stress in STZ-treated type 1 diabetic mice, whereas mTOR hyperactivation due to increased levels of insulin was suggested to cause the autophagy "numbness" in $d b / d b$ type 2 diabetic mice. Our current study does not dismiss the existence of distinct mechanisms of autophagy impairment in type 1 and 2 diabetes; instead, we suggest that there are also common mechanisms between them that may be therapeutic targets. In fact, $\mathrm{DKD}$, including tubulointerstitial damage, in rats was improved by a low-protein diet through restoration of autophagy (22).

Despite the emerging evidence of autophagy impairment in diabetes, the upstream signaling leading to the impairment remains unclear (14-16). We demonstrated the downregulation of several autophagy proteins early during diabetes, along with autophagy impairment. Among them, ULK1 was downregulated in all tested models and in human diabetic kidneys (Figures 5 and 11). ULK1 is a key serine/threonine protein kinase involved in the initiation of autophagy $(12,32)$, and, accordingly, its downregulation in DKD is expected to impair autophagy. Interestingly, ULK1 mRNA levels were not reduced in the kidneys of Akita diabetic mice and were only slightly reduced in STZ-induced diabetic mice, indicating that ULK1 downregulation in DKD does not mainly occur at the transcriptional level. In addition, changes were not detectable for the factors involved in the regulation of ULK1 stability, including Fip200, ATG13, and AMBRA1. These observations led us to investigate microRNAs as a potential mechanism responsible for ULK1 downregulation in DKD.

microRNAs are endogenously produced, short RNAs of 22 to 25 nucleotides that repress target gene expression posttranscriptionally by binding to the 3 '-UTRs of their mRNAs (38). The first microRNA shown to regulate DKD is miR-192, which promotes the production of extracellular matrix (ECM) proteins, collagens, and profibrotic factors $(44,45)$. Subsequent studies have demonstrated important roles of specific microRNAs in DKD that were found to function in various cell types in kidneys $(5,46-48)$. In the current study, bioinformatics analysis suggested that ULK1 is a top target of miR-214. Experimentally, we showed that miR214 was significantly upregulated in diabetic mouse kidneys and HG-treated renal tubule cells. Moreover, blockade of miR-214 with a specific LNA or ablation of miR-214 in kidney proximal tubules prevented the ULK1 decrease in DKD models, which was accompanied by the preservation of autophagy and amelioration of renal hypertrophy and DKD (Figure 7). Further analyses, including a luciferase microRNA target assay, proved that ULK1 is indeed a direct target of miR-214. Also, in human diabetic kidneys, miR-214 and ULK1 expression had an inverse relationship (Figure 12). Together, these results indicate that miR-214 was 
A
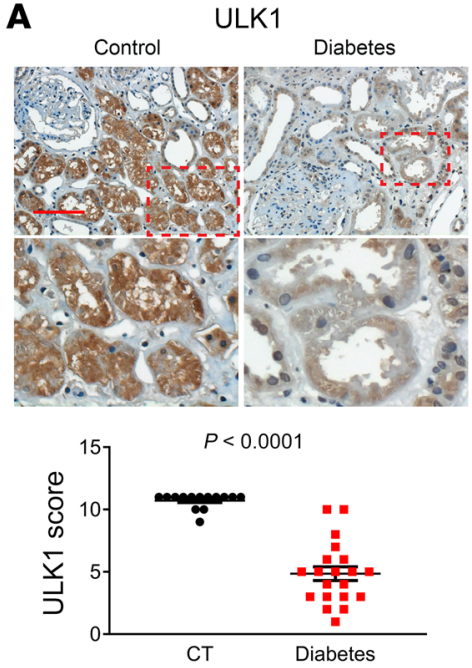

B
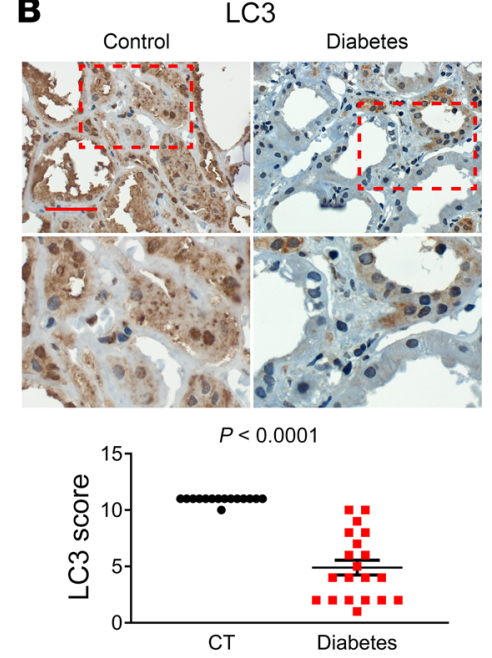

Figure 11. ULK1 downregulation and autophagy impairment in human diabetic kidneys. Renal biopsies from diabetic patients and nondiabetic patients were examined for the expression of ULK1 (A) and LC3 (B) by IHC ( $n=14$ control and $n=20$ diabetic renal biopsies). Scale bars: $0.1 \mathrm{~mm}$. Original magnification, $\times 200$ (insets). Compared with the control, the renal biopsies from patients with diabetes had significantly lower ULK1 and LC3 expression in kidney tubules (2-tailed Student's $t$ test). induced in DKD and repressed ULK1 expression, leading to autophagy impairment. In agreement with our results, other studies reported a significant upregulation of miR-214 in kidney cortex of OVE26 type 1 diabetic mice and $d b / d b$ type 2 diabetic mice, although those studies identified PTEN as a target of miR-214 (49, 50). In addition, genetic deletion or inhibition of miR-214 ameliorated renal fibrosis in the unilateral ureter obstruction model (51). The latest work by Bai et al. further suggested that miR-214 may promote CKD by disrupting mitochondrial oxidative phosphorylation (52). Therefore, depending on the cellular context, miR214 may target multiple genes to regulate renal pathophysiology. The current study identified ULK1 as a critical target of miR-214, and downregulation of ULK1 by miR-214 contributes to renal hypertrophy and related pathologies in the pathogenesis of DKD.

Despite the observation of miR-214 induction in renal disease models, very little was known about the underlying mechanism $(49,50)$. Our current data indicate that p53 is the upstream transcription factor that leads to miR-214 induction in DKD. p53, a well-known tumor suppressor, is a stress response gene in cell biology (53). Upon activation, p53 mediates cellular responses including cell-cycle arrest, senescence, and apoptosis. It is also increasingly recognized that p53 may be a key regulator of autophagy (54). Interestingly, the regulation of autophagy by p53 depends on its subcellular localizations. In the nucleus, p53 promotes autophagy by inducing the transcription of pro-autophagy genes, such as DRAM and sestrins, whereas in the cytoplasm, p53 antagonizes autophagy by mechanisms including the promotion of Beclin1 (Atg6) ubiquitination and degradation (54). In the current study, we showed p53 activation in various models of DKD as well as in human diabetic kidneys. Of note, p53 was mainly localized in the cell nucleus in kidney tissues from diabetic mice and patients (Figure 8A, Figure 10A, and Supplemental Figure 11A). Importantly, genetic deletion or pharmacological inhibition of p53 suppressed miR-214 expression, which was accompanied by the preservation of ULK1 and autophagy in renal tubule cells in DKD. We further showed that p53 may directly bind to the promoter of miR-214 for its transcription. Collectively, these results indicate that p53 may suppress ULK1 and autophagy by inducing miR-214. Therefore, depending on the cellular context, even in the nucleus p53 may play an autophagy-inhibitory role. The pathogenic role of p53 has been demonstrated in acute kidney disease, including ischemic and nephrotoxic acute kidney injury $(55,56)$. In DKD, Natarajan and colleagues demonstrated an interesting feedback amplification circuit between p53 and miR-192 in mesangial cells that contributes to the pathogenesis of DKD (57). In the present study, ablation of $p 53$ from kidney proximal tubules reduced renal hypertrophy and prevented the decline of renal function in DKD (Figure 9), indicating that p53 may have a broader role in DKD by activating distinct pathogenic mechanisms in different kidney cells. The mechanism of p53 activation in diabetic kidneys is currently unclear. We speculate that it may be related to oxidative stress and the DNA damage response. Exposure of mesangial cells (58), podocytes (59), and rat renal tubule cells (60) to HG induced ROS and upregulated p53 expression. In vivo, glomeruli isolated from diabetic mice showed higher $\mathrm{p} 53$ expression than was seen in nondiabetic mice (59). In a recent study, erianin decreased ROS production and p53 expression in HG-treated rat renal tubule cells, implying a role of ROS in p53 activation in this model (60). HG levels also triggered DNA damage through ROS overproduction (61), which may induce p53 activation in diabetes. A thorough investigation will be needed to analyze ROS, the DNA damage response, and $\mathrm{p} 53$ expression in DKD.

Besides renal tubule cells, an autophagy defect may occur in DKD in other kidney cells as well, including glomerular podocytes and mesangial cells. p53 is upregulated in HG-treated podocytes and mesangial cells and also in glomeruli in diabetic animals $(58,59)$. To date, changes in miR-214 expression in HG-treated podocytes have not, to our knowledge, been reported. However, mesangial cells exposed to HG showed augmented expression of miR-214 accompanied by cellular hypertrophy (50). Thus, the findings in the present study may be applicable to podocytes and mesangial cells in $\mathrm{DKD}$, which deserve further investigation.

In summary, using both pharmacological and genetic approaches, we have demonstrated the impairment of tubular autophagy and its role, as well as the underlying mechanism in both DKD models and diabetic human kidneys. Autophagy impairment under this condition was instigated by the downregulation of ULK1 via p53-mediated induction of miR-214. We found that autophagy 

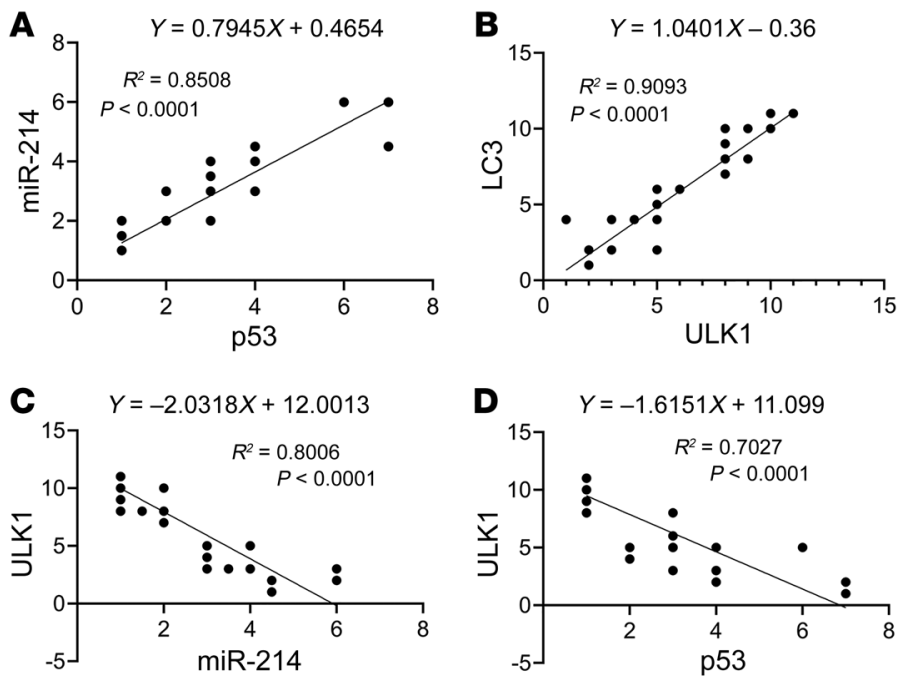

Figure 12. Linear correlation and regression analysis of $p 53$ activation, miR-214 induction, ULK1 downregulation, and autophagy impairment in human diabetic kidneys. Renal biopsies from diabetic patients and nondiabetic patients were examined for the expression of $\mathrm{p} 53$, ULK1, and LC3 by IHC and of miR-214 by ISH ( $n=14$ control and $n=20$ diabetic renal biopsies). Linear correlation and regression analyses were performed, and showed a significant positive correlation between p53 and miR-214 expression (A) and between ULK1 expression and the formation of LC3 puncta (B), whereas a negative correlation was found between miR-214 and ULK1 (C) and between $\mathrm{p} 53$ and ULK1 expression (D) (Spearman's correlation test). impairment exacerbated renal hypertrophy and related pathologies, contributing to renal dysfunction and the progression of DKD, which correlated well with renal fibrosis in patients with diabetes. Identification of the $\mathrm{p} 53 / \mathrm{miR}-214 / \mathrm{ULK} 1$ axis in DKD unveils possible molecular targets for the prevention and treatment of DKD.

\section{Methods}

Animals. C57BL/6J and Akita mice were obtained from The Jackson Laboratory. Kidney proximal tubule-specific Atg7 and p53 geneknockout mouse models were established in our recent studies (31, 41 ), and miR-214-knockout mice were generated by breeding miR214-floxed mice (39) with PEPCK-Cre mice (40). Littermate animals were compared in these gene-knockout mouse models. Genotyping of PT-miR-214 mice was done by PCR using 3 primers ( $5^{\prime}$-TTGAATTCTGACTGCATGAGG-3'; 5'-GGGAAAGGAGAGAAACACACA-3'; and $5^{\prime}$-TCCCTGTGACTGCACTAGAAAA-3') that amplify a $235 \mathrm{bp}$ fragment from the WT allele, a $344 \mathrm{bp}$ fragment from the floxed allele, and a 588 bp fragment from the knockout allele. The RFP-GFP-LC3 autophagy reporter mouse model was established recently (29).

STZ induction of diabetes in mice. Six- to 8-week-old male mice were injected i.p. with $50 \mathrm{mg} / \mathrm{kg} \mathrm{STZ}$ in monohydrate sodium citrate solution, $\mathrm{pH} 4.5$, daily for 5 consecutive days, and control mice were injected with the vehicle solution. Body weight and blood glucose concentration were monitored once before STZ injection and then every 2 weeks thereafter. Mice with a glucose concentration exceeding 280 $\mathrm{mg} / \mathrm{dL}$ were considered diabetic.

LNA-anti-miR-214 treatment. Mice were injected with $50 \mu \mathrm{g}$ LNAanti-miR-214 (Exiqon), scrambled LNA (LNA-anti-miR-214 control), or PBS via retro-orbital injection every 3 weeks for a total of 7 injections. The mice were euthanized 1 week after the final injection for analysis.

Analysis of the ACR. Urinary albumin excretion was determined using Albuwell $\mathrm{M}$ and urinary creatinine using Creatinine Companion kits (Exocell) according to the manufacturer's instructions.

TUNEL assay of cell death. Paraffin-embedded tissue sections were stained with the in situ Cell Death Detection Kit (Roche Applied Science). TUNEL-positive cells were counted from 10 random images for each specimen from the kidney cortex and outer medulla in a blinded manner.
Cells and treatments. The RPTC and BUMPT renal proximal tubule cell lines were obtained, respectively, from U. Hopfer (Case Western Reserve University, Cleveland, Ohio, USA) and Wilfred Lieberthal (Boston University School of Medicine, Boston, Massachusetts, USA), and HEK293 cells were obtained from American Type Culture Collection (ATCC). These cells were cultured in DMEM medium with $10 \%$ FBS. To inhibit miR-214, $200 \mathrm{nM}$ anti-mmu-miR-214 LNAs (Exiqon) were transfected into RPTCs with Lipofectamine 2000 (Thermo Fisher Scientific). To overexpress miR-214 in HEK293 cells, a mature miR-214 plasmid or empty vector from Origene was transfected and selected by $2.5 \mu \mathrm{g} / \mathrm{mL}$ puromycin for 2 weeks to obtain cells with stable miR-214 expression. For HG treatment, cells were seeded to reach $70 \%-80 \%$ confluence. After overnight growth, the cells were subjected to serum-free starvation for 20 hours and then incubated in $30 \mathrm{mM}$ glucose medium, NG (5.5 mM) medium, or medium containing 5.5 $\mathrm{mM}$ glucose and $24.5 \mathrm{mM}$ mannitol (controls).

FACS analysis of cell size change. Cell size change was determined using the cell-cycle analysis kit from GenScript. Briefly, cells were harvested by trypsinization, washed in PBS, and fixed in $70 \%$ ethanol overnight. Subsequently, cells were washed in PBS, treated with RNase for 30 minutes at $37^{\circ} \mathrm{C}$, and stained with propidium iodide for 30 minutes on ice. Forward light scatter was expressed in arbitrary units. Units are expressed as the relative change compared with the control group.

Analysis of autophagy in cells. Autophagy in cultured cells was examined by 2 methods. In the first method, cells were transiently transfected with GFP-LC3. After treatment, the cells were fixed with $4 \%$ paraformaldehyde. Approximately 100 cells with GFP fluorescence from 10-20 random fields (×630 magnification) were analyzed in each condition. The number of GFP-LC3 puncta per cell was determined using ImageJ software (NIH). In the second method, LC3-II was analyzed by immunoblotting. Additionally, autophagic flux was determined by a LC3-II turnover assay, in which the amount of LC3-II was compared in the presence versus absence of $20 \mu \mathrm{M}$ chloroquine.

Analysis of autophagy in kidneys. For analysis of autophagy in reporter mice, autophagosome formation and maturation to autolysosomes was analyzed using CAG-RFP-GFP-LC3-transgenic mice. Following treatment, CAG-RFP-GFP-LC3 mice were perfused with $4 \%$ 
paraformaldehyde in PBS. Kidneys were further fixed overnight, balanced with $30 \%$ sucrose in PBS, and embedded in OCT compound for cryosectioning. Tissue cryosections of $5 \mu \mathrm{m}$ thickness were washed in PBS and mounted with ProLong Diamond Antifade Reagent (Thermo Fisher Scientific). For each section, 8-10 random fields ( $\times 630$ magnification) were selected for counting of green GFP-labeled puncta and red RFP-labeled puncta. Autophagosomes were indicated by GFP dots, and the number of autolysosomes was calculated by subtracting the number of GFP dots from the number of RFP dots. For IHC staining of LC3, mice were perfusion fixed with $4 \%$ paraformaldehyde in PBS for the collection of kidneys for further overnight fixation followed by paraffin embedment. Tissue sections of $5 \mu \mathrm{m}$ thickness were deparaffinized and subjected to antigen retrieval in $1 \mathrm{mM}$ EDTA, $0.05 \%$ Tween-20, $\mathrm{pH} 8.0$, at $95^{\circ} \mathrm{C}-100^{\circ} \mathrm{C}$. The sections were then exposed to $3 \% \mathrm{H}_{2} \mathrm{O}_{2}$ in PBS, blocking buffer containing $2 \%$ BSA, $0.2 \%$ milk, $2 \%$ normal goat serum, and $0.8 \%$ Triton X-100, and anti-LC3 antibody at $4^{\circ} \mathrm{C}$ overnight. Then, the sections were incubated with avidin and biotin, followed by incubation with a biotin-donkey anti-rabbit secondary antibody for 1 hour at room temperature. After further washing, the sections were incubated with TNB (0.1 M Tris- $\mathrm{HCl}, \mathrm{pH} 7.5,0.15 \mathrm{M}$ $\mathrm{NaCl}, 0.5 \%$ blocking reagent) blocking buffer followed by streptavidin-HRP. Signals of the antigen-antibody complexes were developed with a DAB Kit (Vector Laboratories). For electron microscopy analysis of autophagic vacuoles, mice were sacrificed and perfused with $10 \mathrm{~mL}$ (10 units $/ \mathrm{mL}$ ) heparin, followed by $50 \mathrm{~mL}$ fixative $(100 \mathrm{mM}$ sodium cacodylate, $2 \mathrm{mM} \mathrm{CaCl}_{2}, 4 \mathrm{mM} \mathrm{MgSO}_{4}, 4 \%$ paraformaldehyde, and $2.5 \%$ glutaraldehyde). Kidneys were then harvested and postfixed in the same fixative. Tissue cubes of approximately $1 \mathrm{~mm}^{3}$ size were collected from each kidney for standard electron microscopy processing.

IHC and immunofluorescence. Kidney samples were collected and fixed immediately in $4 \%$ paraformaldehyde. Paraffin-embedded sections of kidney tissue ( $4 \mu \mathrm{m}$ or $2 \mu \mathrm{m}$ thick) from mice or patients with diabetes, respectively, were used for IHC. After rehydration, the sections were incubated in blocking buffer containing 2\% BSA, $0.2 \%$ milk, and $2 \%$ normal goat serum in PBS with $0.8 \%$ Triton $\mathrm{X}-100$. Tissue sections were exposed to the following primary antibodies: anti-p53 (catalog 2524, Cell Signaling Technology), anti-fibronectin (ab2413, Abcam), anti-macrophage (ab56297, Abcam), anti-collagen I 1 (NBP1-30054, Novus), and anti-ULK1 (NBP2-24738, Novus) overnight at $4^{\circ} \mathrm{C}$, followed by incubation with an HRP-conjugated secondary antibody (DAKO) or a FITC-goat anti-rabbit secondary antibody (Jackson ImmunoResearch Laboratories). After signal amplification using the TSA Biotin System (PerkinElmer), the slides were developed with a VECTASTAIN Elite ABC Kit and ImmPACT DAB Peroxidase Substrate (Vector Laboratories).

RNA extraction and real-time PCR. Kidney cortical and outer medulla samples were dissected freshly frozen in liquid $\mathrm{N}_{2}$ and kept at $-80^{\circ} \mathrm{C}$ until use. Total RNA was extracted from cells or frozen mouse kidney samples with a mirVana miRNA Isolation Kit (Life Technologies, Thermo Fisher Scientific). For regular real-time quantitative PCR (RTqPCR), $1 \mu \mathrm{g}$ RNA was reverse transcribed using a cDNA Transcription Kit (Bio-Rad), and RT-qPCR was performed using SYBR Green PCR Master Mix (Bio-Rad). $\beta$-Actin was used for normalization. For real-time PCR of microRNA, 40 ng total RNA was converted to cDNA using the TaqMan MicroRNA Reverse Transcription Kit (Life Technologies, Thermo Fisher Scientific). Real-time PCR was performed using TaqMan MicroRNA Assays (Life Technologies, Thermo Fisher Scientific). Small nuclear RNA 202 was used as an internal control, and the quantification was done using $\Delta \mathrm{Ct}$ values as recently described $(62,63)$.

ChIP assay. Cells were fixed with $0.75 \%$ formaldehyde, neutralized with glycine, and collected in cold PBS, followed by centrifugation at $1000 \mathrm{~g}$ for 5 minutes at $4^{\circ} \mathrm{C}$. The cell pellet was resuspended and lysed in sonication buffer (5 mM HEPES, pH 7.9, $140 \mathrm{mM} \mathrm{NaCl}$, $1 \mathrm{mM}$ EDTA, $1 \%$ Triton X-100, 0.1\% Na-deoxycholate, and $0.1 \%$ SDS) for 10 minutes at $4^{\circ} \mathrm{C}$, and then sonicated 6 times for 15 seconds each to shear the DNA. After centrifugation at $8000 \mathrm{~g}$ for 30 seconds at $4^{\circ} \mathrm{C}$, the chromatin samples were collected from the supernatant, and $50 \mu \mathrm{L}$ aliquots of the chromatin samples were used for input DNA extraction. After incubation with $1 \mu \mathrm{L}$ of $1 \mathrm{mg} / \mathrm{mL}$ RNase A (QIAGEN) and $2 \mu \mathrm{L}$ proteinase $\mathrm{K}(20 \mathrm{mg} / \mathrm{mL})$ at $65^{\circ} \mathrm{C}$ overnight, the input DNA was extracted using the QIAquick PCR Purification Kit (QIAGEN). The chromatin samples were then diluted with sonication buffer to 1 or $2 \mathrm{mg} / \mathrm{mL}$ DNA and incubated for 2 hours at $4^{\circ} \mathrm{C}$ with $1 \mathrm{mg} / \mathrm{mL}$ BSA and $40 \mu \mathrm{L}$ protein A agarose beads (Santa Cruz Biotechnology) that were prebalanced with sperm DNA and BSA in $1 \mathrm{~mL}$ reaction buffer. After removing the beads by centrifugation, $4 \mu \mathrm{g}$ anti-p53 antibody was added to the reaction for overnight incubation, followed by precipitation with protein A agarose beads. The beads were washed with sonication buffer, buffer A (50 mM HEPES, pH 7.9, $500 \mathrm{mM} \mathrm{NaCl}$, 1 mM EDTA, $1 \%$ Triton X-100, 0.1\% Na-deoxycholate, and 0.1\% SDS), buffer B (20 mM Tris-HCl, pH 8.0, 1 mM EDTA, $250 \mathrm{mM} \mathrm{LiCl,} \mathrm{0.5 \%}$ NP-40, and $0.5 \%$ Na-deoxycholate), and TE buffer (Tris-EDTA). The DNA was then eluted with $50 \mathrm{mM}$ Tris-HCl, pH 8.0, 1 mM EDTA, 1\% SDS, and $50 \mathrm{mM} \mathrm{NaHCO}_{3}$ and purified with the QIAquick PCR Purification Kit after RNase A treatment. The DNA samples were analyzed by real-time PCR with input DNA for normalization using primers designed according to the p53 binding site sequence in the miR-214 gene (forward, CAAGGCTATGGCACTTACCTAC; reverse, TTTCACAAGACCTCTGCATCTC).

Plasmids and site-directed mutagenesis. For construction of the luciferase reporter plasmid, the full-length 3'-UTR of ULK1 was amplified from human genomic DNA by PCR and inserted into the 3 '-UTR of the luciferase gene of the pMIR-REPORT luciferase plasmid (Life Technologies, Thermo Fisher Scientific) using the ULK1 3'-UTR amplification primers (forward, GGACTAGTGGGTGTCTCCCATCTTTT; reverse, CCCAAGCTTAAAGGGTCCAGCACTAT). The mutated 3'-UTR luciferase reporter plasmids were generated by site-directed mutagenesis using the Q5 Site-Directed Mutagenesis Kit (New England BioLabs) according to the manufacturer's instructions. The miR-214 target sequence for human ULK1 was mutant from CTGCTG to ATATAT with the following primers: forward, TCACCAGGGAATATATGGCAGCGATTC; reverse, GGTCCAGGTTCTGTCTCTATATG.

Luciferase reporter assay of the microRNA target. The PMIR-REPORT miRNA Expression Reporter Vector System was used for the luciferase reporter assay. For analysis of luciferase activity in miR-214-overexpressed RPTCs, the plasmids with or without the ULK1 3'-UTR insert were cotransfected into RPTCs with the pMIR-REPORT $\beta$-gal Control Plasmid (Life Technologies, Thermo Fisher Scientific) and the miR-214 expression plasmid or empty vector (Origene). One day after the transfection, cell lysate was collected in reporter lysis buffer. Luciferase activity was measured using the Luciferase Assay System (Promega), and $\beta$-galactosidase activity was measured using the $\beta$-Galactosidase Enzyme Assay System (Promega). Luciferase activity was normalized to $\beta$-galactosidase activity. 
Immunoblot analysis. Cells or freshly frozen tissues were lysed with $2 \%$ SDS in lysis buffer (62.5 mM Tris- $\mathrm{HCl}, \mathrm{pH} 6.8,10 \%$ glycerol) to determine the protein concentration using a Pierce BCA Protein Assay Kit (Thermo Fisher Scientific). Equal amounts of proteins were resolved by SDS-PAGE and were subjected to immunoblot analysis using standard methods with the following antibodies: ULK1 (a7481, 1:1000), Atg7 (A2856, 1:200), actin (A5441, 1:10,000), and cyclophilin B (SAB4200201, 1:5000) (all from MilliporeSigma); Atg5 (ab54032, 1:1000, Abcam); Beclin1 (sc-11427, 1L1000, Santa Cruz Biotechnology); p-ULK1 (Ser757) (6888, 1:1000), p-ULK1 (Ser555) (5869, 1:1000), p-P70S6K (Thr389) (9234, 1:1000), P70S6K (2708, 1:1000), GAPDH (2118, 1:5000), p-p53 (Ser15) (9284,1:1000), and p53 (2524, 1:1000) (all from Cell Signaling Technology); Atg13 (18258-1-AP, 1:1000, Proteintech); LC3 (NB100-2220, 1:1000) and AMBRA1 (2619002, 1:1000) (both from Novus); and Fip200 (07-1815, 1:1000) and p-AMBRA1 (ABC80, 1:1000) (both from MilliporeSigma). For densitometric analysis, pixel intensity was quantified using ImageJ software.

Human kidney tissues and analysis. Renal biopsies from 20 diabetic patients and 14 nondiabetic patients were obtained from the Department of Nephrology of Shanghai Changzheng Hospital at the Second Military Medical University (Shanghai, China). The nondiabetic samples were peritumoral, normal kidney tissues. The tissues were formalin fixed, paraffin embedded, and sectioned at $2 \mu \mathrm{m}$ thickness for IHC staining of p53, ULK1, and LC3, or ISH analysis of miR-214. For quantitative analysis of ULK1 and LC3 expression, the renal tubules with reduced staining were counted from 5 to 10 images ( $\times 200$ magnification) in each specimen, and the percentage of tubules with reduced staining was calculated. The score for ULK1 and LC3 staining was determined according to the percentage of renal tubules showing reduced staining: 11 , no reduction in staining; $10,<10 \% ; 9,10 \%-20 \%$; 8, 20\%-30\%; 7, 30\%-40\%; 6, 40\%-50\%; 5, 50\%-60\%; 4, 60\%-70\%; 3, 70\%-80\%; 2, 80\%-90\%; 1, >90\%.p53-positive tubules were counted in 5 to 10 images ( $\times 200$ magnification) for each specimen. The histological score for p53 staining was determined on the basis of the average number of $\mathrm{p} 53$-positive tubules as follows: 1 , no positive tubules; 2,1 to $3 ; 3,4$ to $6 ; 4,7$ to $9 ; 5,10$ to $12 ; 6,13$ to $15 ; 7,16$ to 18 ; and 8,19 to 21 positive tubules, respectively. For quantification of miR214 expression in ISH, the percentage of miR-214-positive tubules in 5 to 10 images ( $\times 200$ magnification) for each specimen was calculated. miR-214 expression was scored as follows: 1 , no staining; $2,<20 \% ; 3$, $20 \%-40 \% ; 4,40 \%-60 \% ; 5,60 \%-80 \% ; 6$, >80\%. Clinical pathology, including IFTA, interstitial inflammation, and vascular lesions, was analyzed according to the guidelines for the pathological classification of diabetic nephropathy (42). Renal fibrosis was examined by Sirius Red/Fast Green Collagen staining (Chondrex), and quantification was performed using Image J software.

Statistics. Potential p53 binding sites of miR-214 were analyzed with the JASPAR database (http://jaspar.genereg.net). The potential mRNA targets of miR-214 were analyzed with the miRanda database (http://www.microrna.org). For statistical analysis, GraphPad Prism 7.03 was used for ANOVA, and Microsoft Excel 2012 was used for all other calculations. Differences between 2 groups were assessed using a 2-tailed Student's $t$ test. Differences among more than 2 groups were assessed by 1-way ANOVA. Linear regression analysis was performed using Microsoft Excel 2012. Data are expressed as the mean \pm SD. $P$ values of less than 0.05 were considered significant.

Study approval. Collection of human kidney tissue was approved by the ethics committee of the Second Military Medical University (Shanghai, China). Informed consent was obtained from all subjects. Animal studies were approved by the IACUC of Charlie Norwood Veterans Affairs Medical Center (Augusta, Georgia, USA.

\section{Author contributions}

ZM and ZD designed the study. ZM, LL, MJL, and DZ performed experiments. ZM, MJL, QM, MZ, HFD, YH, CM, and ZD performed data analysis and interpretation and wrote the manuscript.

\section{Acknowledgments}

We thank Eric Olson and Joseph Hill at The University of Texas Southwestern Medical Center (Dallas, Texas, USA) for providing miR-214-floxed mice and CAG-RFP-GFP-LC3-transgenic mice, respectively. We also thank Volker Haase at Vanderbilt University School of Medicine (Nashville, Tennessee, USA) for providing the PEPCK-Cre mouse line. This study was supported in part by grants from the US Department of Veterans Affairs (Merit Review Award I01 BX000319) and the NIH (DK058831 and DK087843). $\mathrm{ZD}$ is a recipient of a Senior Research Career Scientist award from the US Department of Veterans Affairs.

Address correspondence to: Zheng Dong, Department of Cellular Biology and Anatomy, Medical College of Georgia at Augusta University and Charlie Norwood VA Medical Center, 1459 Laney Walker Blvd., Augusta, Georgia 30912, USA. Phone:706.721.2825; Email: zdong@augusta.edu.
1. Bonventre JV. Can we target tubular damage to prevent renal function decline in diabetes? Semin Nephrol. 2012;32(5):452-462.

2. Kanwar YS, Sun L, Xie P, Liu FY, Chen S. A glimpse of various pathogenetic mechanisms of diabetic nephropathy. Annu Rev Pathol. 2011;6:395-423.

3. Tang SC, Lai KN. The pathogenic role of the renal proximal tubular cell in diabetic nephropathy. Nephrol Dial Transplant. 2012;27(8):3049-3056.

4. Vallon V, Thomson SC. Renal function in diabetic disease models: the tubular system in the pathophysiology of the diabetic kidney. Annu Rev Physiol. 2012;74:351-375.

5. Badal SS, Danesh FR. New insights into molecular mechanisms of diabetic kidney disease. $A m J$
Kidney Dis. 2014;63(2 Suppl 2):S63-S83.

6. Reidy K, Kang HM, Hostetter T, Susztak K. Molecular mechanisms of diabetic kidney disease. J Clin Invest. 2014;124(6):2333-2340.

7. Sharma K. Mitochondrial hormesis and diabetic complications. Diabetes. 2015;64(3):663-672.

8. Kato M, Natarajan R. Epigenetics and epigenomics in diabetic kidney disease and metabolic memory. Nat Rev Nephrol. 2019;15(6):327-345.

9. Satriano J. Kidney growth, hypertrophy and the unifying mechanism of diabetic complications. Amino Acids. 2007;33(2):331-339.

10. Wolf G, Ziyadeh FN. Molecular mechanisms of diabetic renal hypertrophy. Kidney Int. 1999;56(2):393-405.

11. Rigalleau V, et al. Large kidneys predict poor renal outcome in subjects with diabetes and chronic kidney disease. BMC Nephrol. 2010;11:3.

12. Galluzzi L, et al. Molecular definitions of autophagy and related processes. EMBO J. 2017;36(13):1811-1836.

13. Mizushima N, Levine B, Cuervo AM, Klionsky DJ. Autophagy fights disease through cellular selfdigestion. Nature. 2008;451(7182):1069-1075.

14. Ding Y, Choi ME. Autophagy in diabetic nephropathy. J Endocrinol. 2015;224(1):R15-R30.

15. Yang D, et al. Autophagy in diabetic kidney disease: regulation, pathological role and therapeutic potential. Cell Mol Life Sci. 2018;75(4):669-688.

16. Kume S, Thomas MC, Koya D. Nutrient sensing, autophagy, and diabetic nephropathy. Diabetes. 2012;61(1):23-29. 
17. Fang L, et al. Autophagy attenuates diabetic glomerular damage through protection of hyperglycemia-induced podocyte injury. PLOS ONE. 2013;8(4):e60546.

18. Tagawa A, et al. Impaired podocyte autophagy exacerbates proteinuria in diabetic nephropathy. Diabetes. 2016;65(3):755-767.

19. Barbosa Júnior Ade A, Zhou H, Hültenschmidt D, Totovic V, Jurilj N, Pfeifer U. Inhibition of cellular autophagy in proximal tubular cells of the kidney in streptozotocin-diabetic and uninephrectomized rats. Virchows Arch, B, Cell Pathol. 1992;61(6):359-366.

20. Yamahara K, et al. Obesity-mediated autophagy insufficiency exacerbates proteinuria-induced tubulointerstitial lesions. J Am Soc Nephrol. 2013;24(11):1769-1781.

21. Kitada M, Takeda A, Nagai T, Ito H, Kanasaki K, Koya D. Dietary restriction ameliorates diabetic nephropathy through anti-inflammatory effects and regulation of the autophagy via restoration of Sirt1 in diabetic Wistar fatty (fa/fa) rats: a model of type 2 diabetes. Exp Diabetes Res. 2011;2011:908185.

22. Kitada M, et al. A very-low-protein diet ameliorates advanced diabetic nephropathy through autophagy induction by suppression of the mTORC1 pathway in Wistar fatty rats, an animal model of type 2 diabetes and obesity. Diabetologia. 2016;59(6):1307-1317.

23. Sakai S, et al. Proximal tubule autophagy differs in type 1 and 2 diabetes. J Am Soc Nephrol. 2019;30(6):929-945.

24. Huang C, et al. Thioredoxin interacting protein (TXNIP) regulates tubular autophagy and mitophagy in diabetic nephropathy through the mTOR signaling pathway. Sci Rep. 2016;6:29196.

25. Liu WJ, et al. Autophagy-lysosome pathway in renal tubular epithelial cells is disrupted by advanced glycation end products in diabetic nephropathy. J Biol Chem. 2015;290(33):20499-20510.

26. Xu Y, et al. The renoprotective role of autophagy activation in proximal tubular epithelial cells in diabetic nephropathy. J Diabetes Complicat. 2015;29(8):976-983.

27. Brosius FC, et al. Mouse models of diabetic nephropathy. J Am Soc Nephrol. 2009;20(12):2503-2512.

28. Azushima K, Gurley SB, Coffman TM. Modelling diabetic nephropathy in mice. Nat Rev Nephrol. 2018;14(1):48-56.

29. Li L, Wang ZV, Hill JA, Lin F. New autophagy reporter mice reveal dynamics of proximal tubular autophagy. J Am Soc Nephrol. 2014;25(2):305-315.

30. Livingston MJ, Ding HF, Huang S, Hill JA, Yin XM, Dong Z. Persistent activation of autophagy in kidney tubular cells promotes renal interstitial fibrosis during unilateral ureteral obstruction. Autophagy. 2016;12(6):976-998.

31. Jiang M, Wei Q, Dong G, Komatsu M, Su Y,
Dong Z. Autophagy in proximal tubules protects against acute kidney injury. Kidney Int . 2012;82(12):1271-1283.

32. Mizushima N. The role of the Atg1/ULK1 complex in autophagy regulation. Curr Opin Cell Biol. 2010;22(2):132-139.

33. Kim J, Kundu M, Viollet B, Guan KL. AMPK and $\mathrm{mTOR}$ regulate autophagy through direct phosphorylation of Ulk1. Nat Cell Biol. 2011;13(2):132-141.

34. Ganley IG, Lam du H, Wang J, Ding X, Chen S, Jiang X. ULK1.ATG13.FIP200 complex mediates mTOR signaling and is essential for autophagy. J Biol Chem. 2009;284(18):12297-12305.

35. Jung CH, et al. ULK-Atg13-FIP200 complexes mediate mTOR signaling to the autophagy machinery. Mol Biol Cell. 2009;20(7):1992-2003.

36. Hara T, et al. FIP200, a ULK-interacting protein, is required for autophagosome formation in mammalian cells. J Cell Biol. 2008;181(3):497-510.

37. Nazio F, et al. mTOR inhibits autophagy by controlling ULK1 ubiquitylation, self-association and function through AMBRA1 and TRAF6. Nat Cell Biol. 2013;15(4):406-416.

38. Bartel DP. MicroRNAs: target recognition and regulatory functions. Cell. 2009;136(2):215-233.

39. Aurora AB, et al. MicroRNA-214 protects the mouse heart from ischemic injury by controlling $\mathrm{Ca}^{2+}$ overload and cell death. J Clin Invest. 2012;122(4):1222-1232.

40. Rankin EB, Tomaszewski JE, Haase VH. Renal cyst development in mice with conditional inactivation of the von Hippel-Lindau tumor suppressor. Cancer Res. 2006;66(5):2576-2583.

41. Zhang D, et al. Tubular p53 regulates multiple genes to mediate AKI. JAm Soc Nephrol. 2014;25(10):2278-2289.

42. Tervaert TW, et al. Pathologic classification of diabetic nephropathy. J Am Soc Nephrol. 2010;21(4):556-563.

43. Takahashi A, et al. Autophagy inhibits the accumulation of advanced glycation end products by promoting lysosomal biogenesis and function in the kidney proximal tubules. Diabetes. 2017;66(5):1359-1372.

44. Kato M, et al. MicroRNA-192 in diabetic kidney glomeruli and its function in TGF-betainduced collagen expression via inhibition of E-box repressors. Proc Natl Acad Sci USA. 2007;104(9):3432-3437.

45. Putta S, Lanting L, Sun G, Lawson G, Kato M, Natarajan R. Inhibiting microRNA-192 ameliorates renal fibrosis in diabetic nephropathy. J Am Soc Nephrol. 2012;23(3):458-469.

46. Kato M, Natarajan R. Diabetic nephropathy--emerging epigenetic mechanisms. Nat Rev Nephrol. 2014;10(9):517-530.

47. Trionfini P, Benigni A, Remuzzi G. MicroRNAs in kidney physiology and disease. Nat Rev Nephrol. 2015;11(1):23-33.

48. Kantharidis P, Wang B, Carew RM, Lan HY. Dia- betes complications: the microRNA perspective. Diabetes. 2011;60(7):1832-1837.

49. Wang X, et al. Cross talk between miR-214 and PTEN attenuates glomerular hypertrophy under diabetic conditions. Sci Rep. 2016;6:31506.

50. Bera A, Das F, Ghosh-Choudhury N, Mariappan MM, Kasinath BS, Ghosh Choudhury G. Reciprocal regulation of miR-214 and PTEN by high glucose regulates renal glomerular mesangial and proximal tubular epithelial cell hypertrophy and matrix expansion. Am J Physiol, Cell Physiol. 2017;313(4):C430-C447.

51. Denby L, et al. MicroRNA-214 antagonism protects against renal fibrosis. J Am Soc Nephrol. 2014;25(1):65-80.

52. Bai M, et al. MicroRNA-214 promotes chronic kidney disease by disrupting mitochondrial oxidative phosphorylation. Kidney Int. 2019;95(6):1389-1404

53. Murray-Zmijewski F, Slee EA, Lu X. A complex barcode underlies the heterogeneous response of p53 to stress. Nat Rev Mol Cell Biol. 2008;9(9):702-712.

54. Maiuri MC, Galluzzi L, Morselli E, Kepp O, Malik SA, Kroemer G. Autophagy regulation by $\mathrm{p} 53$. Curr Opin Cell Biol. 2010;22(2):181-185.

55. Dagher PC. Apoptosis in ischemic renal injury: roles of GTP depletion and p53. Kidney Int 2004;66(2):506-509.

56. Tang C, et al. P53 in kidney injury and repair: mechanism and therapeutic potentials. Pharma col Ther. 2019;195:5-12.

57. Deshpande SD, et al. Transforming growth factor- $\beta$-induced cross talk between p 53 and a microRNA in the pathogenesis of diabetic nephropathy. Diabetes. 2013;62(9):3151-3162.

58. Lu Q, et al. The mTOR promotes oxidative stress-induced apoptosis of mesangial cells in diabetic nephropathy. Mol Cell Endocrinol. 2018;473:31-43.

59. Eid AA, et al. AMP-activated protein kinase (AMPK) negatively regulates Nox4-dependent activation of p53 and epithelial cell apoptosis in diabetes. J Biol Chem. 2010;285(48):37503-37512.

60. Chen MF, Liou SS, Kao ST, Liu IM. Erianin protects against high glucose-induced oxidative injury in renal tubular epithelial cells. Food Chem Toxicol. 2019;126:97-105.

61. Wang K, et al. DSePA antagonizes high glucoseinduced neurotoxicity: evidences for DNA damage-mediated p53 phosphorylation and MAPKs and AKT pathways. Mol Neurobiol. 2016;53(7):4363-4374

62. Wei Q, et al. MicroRNA-668 represses MTP18 to preserve mitochondrial dynamics in ischemic acute kidney injury. J Clin Invest. 2018;128(12):5448-5464.

63. Bhatt K, et al. MicroRNA- 687 induced by hypoxiainducible factor- 1 targets phosphatase and tensin homolog in renal ischemia-reperfusioninjury. JAm Soc Nephrol. 2015;26(7):1588-1596. 